Article

\title{
Optimal Fourth, Eighth and Sixteenth Order Methods by Using Divided Difference Techniques and Their Basins of Attraction and Its Application
}

\author{
Yanlin Tao ${ }^{1,+}$ and Kalyanasundaram Madhu $2, *,+(D)$ \\ 1 School of Computer Science and Engineering, Qujing Normal University, Qujing 655011, China; \\ taoyanl@126.com \\ 2 Department of Mathematics, Saveetha Engineering College, Chennai 602105, India \\ * Correspondence: kalyan742@pec.edu; Tel.: +91-9840050042 \\ + These authors contributed equally to this work.
}

Received: 26 February 2019; Accepted: 26 March 2019; Published: 30 March 2019

\begin{abstract}
The principal objective of this work is to propose a fourth, eighth and sixteenth order scheme for solving a nonlinear equation. In terms of computational cost, per iteration, the fourth order method uses two evaluations of the function and one evaluation of the first derivative; the eighth order method uses three evaluations of the function and one evaluation of the first derivative; and sixteenth order method uses four evaluations of the function and one evaluation of the first derivative. So these all the methods have satisfied the Kung-Traub optimality conjecture. In addition, the theoretical convergence properties of our schemes are fully explored with the help of the main theorem that demonstrates the convergence order. The performance and effectiveness of our optimal iteration functions are compared with the existing competitors on some standard academic problems. The conjugacy maps of the presented method and other existing eighth order methods are discussed, and their basins of attraction are also given to demonstrate their dynamical behavior in the complex plane. We apply the new scheme to find the optimal launch angle in a projectile motion problem and Planck's radiation law problem as an application.
\end{abstract}

Keywords: non-linear equation; basins of attraction; optimal order; higher order method; computational order of convergence

MSC: 65H05, 65D05, 41A25

\section{Introduction}

One of the most frequent problems in engineering, scientific computing and applied mathematics, in general, is the problem of solving a nonlinear equation $f(x)=0$. In most of the cases, whenever real problems are faced, such as weather forecasting, accurate positioning of satellite systems in the desired orbit, measurement of earthquake magnitudes and other high-level engineering problems, only approximate solutions may get resolved. However, only in rare cases, it is possible to solve the governing equations exactly. The most familiar method of solving non linear equation is Newton's iteration method. The local order of convergence of Newton's method is two and it is an optimal method with two function evaluations per iterative step.

In the past decade, several higher order iterative methods have been developed and analyzed for solving nonlinear equations that improve classical methods such as Newton's method, Chebyshev method, Halley's iteration method, etc. As the order of convergence increases, so does the number of function evaluations per step. Hence, a new index to determine the efficiency called the efficiency index is introduced in [1] to measure the balance between these quantities. Kung-Traub [2] conjectured 
that the order of convergence of any multi-point without memory method with $d$ function evaluations cannot exceed the bound $2^{d-1}$, the optimal order. Thus the optimal order for three evaluations per iteration would be four, four evaluations per iteration would be eight, and so on. Recently, some fourth and eighth order optimal iterative methods have been developed (see [3-14] and references therein). A more extensive list of references as well as a survey on the progress made in the class of multi-point methods is found in the recent book by Petkovic et al. [11].

This paper is organized as follows. An optimal fourth, eighth and sixteenth order methods are developed by using divided difference techniques in Section 2. In Section 3, convergence order is analyzed. In Section 4, tested numerical examples to compare the proposed methods with other known optimal methods. The problem of Projectile motion is discussed in Section 5 where the presented methods are applied on this problem with some existing ones. In Section 6, we obtain the conjugacy maps of these methods to make a comparison from dynamical point of view. In Section 7 , the proposed methods are studied in the complex plane using basins of attraction. Section 8 gives concluding remarks.

\section{Design of an Optimal Fourth, Eighth and Sixteenth Order Methods}

Definition 1 ([15]). If the sequence $\left\{x_{n}\right\}$ tends to a limit $x^{*}$ in such a way that

$$
\lim _{n \rightarrow \infty} \frac{x_{n+1}-x^{*}}{\left(x_{n}-x^{*}\right)^{p}}=C
$$

for $p \geq 1$, then the order of convergence of the sequence is said to be $p$, and $C$ is known as the asymptotic error constant. If $p=1, p=2$ or $p=3$, the convergence is said to be linear, quadratic or cubic, respectively.

Let $e_{n}=x_{n}-x^{*}$, then the relation

$$
e_{n+1}=C e_{n}^{p}+O\left(e_{n}^{p+1}\right)=O\left(e_{n}^{p}\right) .
$$

is called the error equation. The value of $p$ is called the order of convergence of the method.

Definition 2 ([1]). The Efficiency Index is given by

$$
E I=p^{\frac{1}{d}}
$$

where $d$ is the total number of new function evaluations (the values of $f$ and its derivatives) per iteration.

Let $x_{n+1}=\psi\left(x_{n}\right)$ define an Iterative Function (IF). Let $x_{n+1}$ be determined by new information at $x_{n}, \phi_{1}\left(x_{n}\right), \ldots, \phi_{\mathrm{i}}\left(x_{n}\right), \mathrm{i} \geq 1$. No old information is reused. Thus,

$$
x_{n+1}=\psi\left(x_{n}, \phi_{1}\left(x_{n}\right), \ldots, \phi_{\mathrm{i}}\left(x_{n}\right)\right) .
$$

Then $\psi$ is called a multipoint IF without memory.

The Newton (also called Newton-Raphson) IF $\left(2^{\text {nd }} N R\right)$ is given by

$$
\psi_{2^{n d} N R}(x)=x-\frac{f(x)}{f^{\prime}(x)} .
$$

The $2^{\text {nd }} N R$ IF is one-point IF with two function evaluations and it satisfies the Kung-Traub conjecture with $d=2$. Further, $E I_{2^{n d} N R}=1.414$. 


\subsection{An Optimal Fourth Order Method}

We attempt to get a new optimal fourth order IF as follows, let us consider two step Newton's method

$$
\psi_{4^{t h} N R}(x)=\psi_{2^{n d} N R}(x)-\frac{f\left(\psi_{2^{n d} N R}(x)\right)}{f^{\prime}\left(\psi_{2^{\text {nd }} N R}(x)\right)} .
$$

The above one is having fourth order convergence with four function evaluations. But, this is not an optimal method. To get an optimal, need to reduce a function and preserve the same convergence order, and so we estimate $f^{\prime}\left(\psi_{2^{n d} N R}(x)\right)$ by the following polynomial

$$
q(t)=a_{0}+a_{1}(t-x)+a_{2}(t-x)^{2}
$$

which satisfies

$$
q(x)=f(x), q^{\prime}(x)=f^{\prime}(x), q\left(\psi_{2^{n d} N R}(x)\right)=f\left(\psi_{2^{n d} N R}(x)\right)
$$

On implementing the above conditions on Equation (6), we obtain three unknowns $a_{0}, a_{1}$ and $a_{2}$. Let us define the divided differences

$$
f[y, x]=\frac{f(y)-f(x)}{y-x}, f[y, x, x]=\frac{f[y, x]-f^{\prime}(x)}{y-x} .
$$

From conditions, we get $a_{0}=f(x), a_{1}=f^{\prime}(x)$ and $a_{2}=f\left[\psi_{2^{n d} N R}(x), x, x\right]$, respectively, by using divided difference techniques. Now, we have the estimation

$$
f^{\prime}\left(\psi_{2^{n d} N R}(x)\right) \approx q^{\prime}\left(\psi_{2^{n d} N R}(x)\right)=a_{1}+2 a_{2}\left(\psi_{2^{t h} N R}(x)-x\right) .
$$

Finally, we propose a new optimal fourth order method as

$$
\psi_{4^{t h} Y M}(x)=\psi_{2^{n d} N R}(x)-\frac{f\left(\psi_{2^{n d} N R}(x)\right)}{f^{\prime}(x)+2 f\left[\psi_{2^{n d}}(x), x, x\right]\left(\psi_{2^{t h} N R}(x)-x\right)} .
$$

The efficiency of the method (7) is $E I_{4 t h Y M}=1.587$.

\subsection{An Optimal Eighth Order Method}

Next, we attempt to get a new optimal eighth order IF as following way

$$
\psi_{8^{\text {th }} Y M}(x)=\psi_{4^{\text {th }} Y M}(x)-\frac{f\left(\psi_{4^{\text {th }} Y M}(x)\right)}{f^{\prime}\left(\psi_{4^{\text {th }} Y M}(x)\right)} .
$$

The above one is having eighth order convergence with five function evaluations. But, this is not an optimal method. To get an optimal, need to reduce a function and preserve the same convergence order, and so we estimate $f^{\prime}\left(\psi_{4^{\text {th }} Y M}(x)\right)$ by the following polynomial

$$
q(t)=b_{0}+b_{1}(t-x)+b_{2}(t-x)^{2}+b_{3}(t-x)^{3},
$$

which satisfies

$$
q(x)=f(x), q^{\prime}(x)=f^{\prime}(x), q\left(\psi_{2^{\text {nd } N R}}(x)\right)=f\left(\psi_{2^{\text {nd } N R}}(x)\right), q\left(\psi_{4^{\text {th }} Y M}(x)\right)=f\left(\psi_{4^{\text {th }} Y M}(x)\right) .
$$

On implementing the above conditions on (8), we obtain four linear equations with four unknowns $b_{0}, b_{1}, b_{2}$ and $b_{3}$. From conditions, we get $b_{0}=f(x)$ and $b_{1}=f^{\prime}(x)$. To find $b_{2}$ and $b_{3}$, we solve the following equations:

$$
\begin{aligned}
& f\left(\psi_{2^{n d} N R}(x)\right)=f(x)+f^{\prime}(x)\left(\psi_{2^{n d} N R}(x)-x\right)+b_{2}\left(\psi_{2^{n d} N R}(x)-x\right)^{2}+b_{3}\left(\psi_{2^{n d} N R}(x)-x\right)^{3} \\
& f\left(\psi_{4^{\text {th }} Y M}(x)\right)=f(x)+f^{\prime}(x)\left(\psi_{4^{\text {th }} Y M}(x)-x\right)+b_{2}\left(\psi_{4^{\text {th }} Y M}(x)-x\right)^{2}+b_{3}\left(\psi_{4^{\text {th }} Y M}(x)-x\right)^{3} .
\end{aligned}
$$


Thus by applying divided differences, the above equations simplifies to

$$
\begin{aligned}
& b_{2}+b_{3}\left(\psi_{2^{\text {nd }} N R}(x)-x\right)=f\left[\psi_{2^{\text {nd }} N R}(x), x, x\right] \\
& b_{2}+b_{3}\left(\psi_{4^{\text {th }} Y M}(x)-x\right)=f\left[\psi_{4^{\text {th }} Y M}(x), x, x\right]
\end{aligned}
$$

Solving Equations (9) and (14), we have

$$
\begin{aligned}
& b_{2}=\frac{f\left[\psi_{2^{n d} N R}(x), x, x\right]\left(\psi_{4^{\text {th }} P M}(x)-x\right)-f\left[\psi_{4^{\text {th }} Y M}(x), x, x\right]\left(\psi_{2^{n d} N R}(x)-x\right)}{\psi_{4^{\text {th }} Y M}(x)-\psi_{2^{\text {nd }} N R}(x)}, \\
& b_{3}=\frac{f\left[\psi_{4^{\text {th }} Y M}(x), x, x\right]-f\left[\psi_{2^{\text {nd }} N R}(x), x, x\right]}{\psi_{4^{\text {th }} Y M}(x)-\psi_{2^{\text {nd }} N R}(x)} .
\end{aligned}
$$

Further, using Equation (11), we have the estimation

$$
f^{\prime}\left(\psi_{4^{\text {th }} Y M}(x)\right) \approx q^{\prime}\left(\psi_{4^{\text {th }} Y M}(x)\right)=b_{1}+2 b_{2}\left(\psi_{4^{\text {th }} Y M}(x)-x\right)+3 b_{3}\left(\psi_{4^{\text {th }} Y M}(x)-x\right)^{2} .
$$

Finally, we propose a new optimal eighth order method as

$$
\psi_{8^{\text {th }} Y M}(x)=\psi_{4^{\text {th }} \Upsilon M}(x)-\frac{f\left(\psi_{4^{\text {th }} Y M}(x)\right)}{f^{\prime}(x)+2 b_{2}\left(\psi_{4^{\text {th }} \Upsilon M}(x)-x\right)+3 b_{3}\left(\psi_{4^{\text {th }} Y M}(x)-x\right)^{2}} .
$$

The efficiency of the method (12) is $E I_{8 t h Y M}=1.682$. Remark that the method is seems a particular case of the method of Khan et al. [16], they used weight function to develop their methods. Whereas we used finite difference scheme to develop proposed methods. We can say the methods $4^{\text {th }} Y M$ and $8^{\text {th }} Y M$ are reconstructed of Khan et al. [16] methods.

\subsection{An Optimal Sixteenth Order Method}

Next, we attempt to get a new optimal sixteenth order IF as following way

$$
\psi_{16^{\text {th } Y M}}(x)=\psi_{8^{\text {th }} Y M}(x)-\frac{f\left(\psi_{8^{\text {th }} Y M}(x)\right)}{f^{\prime}\left(\psi_{8^{\text {th }} Y M}(x)\right)} .
$$

The above one is having eighth order convergence with five function evaluations. However, this is not an optimal method. To get an optimal, need to reduce a function and preserve the same convergence order, and so we estimate $f^{\prime}\left(\psi_{8^{t h} Y M}(x)\right)$ by the following polynomial

$$
q(t)=c_{0}+c_{1}(t-x)+c_{2}(t-x)^{2}+c_{3}(t-x)^{3}+c_{4}(t-x)^{4},
$$

which satisfies

$$
\begin{gathered}
q(x)=f(x), q^{\prime}(x)=f^{\prime}(x), q\left(\psi_{2^{\text {nd }} N R}(x)\right)=f\left(\psi_{2^{n d} N R}(x)\right), \\
q\left(\psi_{4^{\text {th }} Y M}(x)\right)=f\left(\psi_{4^{\text {th }} Y M}(x)\right), q\left(\psi_{8^{\text {th }} Y M}(x)\right)=f\left(\psi_{8^{\text {th }} Y M}(x)\right) .
\end{gathered}
$$

On implementing the above conditions on (13), we obtain four linear equations with four unknowns $c_{0}, c_{1}, c_{2}$ and $c_{3}$. From conditions, we get $c_{0}=f(x)$ and $c_{1}=f^{\prime}(x)$. To find $c_{2}, c_{3}$ and $c_{4}$, we solve the following equations:

$$
\begin{aligned}
& f\left(\psi_{2^{n d} N R}(x)\right)=f(x)+f^{\prime}(x)\left(\psi_{2^{n d} N R}(x)-x\right)+c_{2}\left(\psi_{2^{n d} N R}(x)-x\right)^{2}+c_{3}\left(\psi_{2^{n d}} N R(x)-x\right)^{3}+c_{4}\left(\psi_{2^{n d} N R}(x)-x\right)^{4} \\
& f\left(\psi_{4^{\text {th }} Y M}(x)\right)=f(x)+f^{\prime}(x)\left(\psi_{4^{\text {th }} Y M}(x)-x\right)+c_{2}\left(\psi_{4^{\text {th }} Y M}(x)-x\right)^{2}+c_{3}\left(\psi_{4^{\text {th }} Y M}(x)-x\right)^{3}+c_{4}\left(\psi_{4^{\text {th }} Y M}(x)-x\right)^{4} \\
& f\left(\psi_{8^{\text {th }} Y M}(x)\right)=f(x)+f^{\prime}(x)\left(\psi_{8^{\text {th }} Y M}(x)-x\right)+c_{2}\left(\psi_{8^{\text {th }} Y M}(x)-x\right)^{2}+c_{3}\left(\psi_{8^{\text {th }} Y M}(x)-x\right)^{3}+c_{4}\left(\psi_{8^{\text {th }} Y M}(x)-x\right)^{4} .
\end{aligned}
$$


Thus by applying divided differences, the above equations simplifies to

$$
\begin{array}{ll}
c_{2}+c_{3}\left(\psi_{2^{\text {nd }} N R}(x)-x\right)+c_{4}\left(\psi_{2^{\text {nd }} N R}(x)-x\right)^{2}=f\left[\psi_{2^{\text {nd }} N R}(x), x, x\right] \\
c_{2}+c_{3}\left(\psi_{4^{\text {th }} Y M}(x)-x\right)+c_{4}\left(\psi_{4^{\text {th }} Y M}(x)-x\right)^{2}=f\left[\psi_{4^{\text {th }} Y M}(x), x, x\right] \\
c_{2}+c_{3}\left(\psi_{8^{\text {th }} Y M}(x)-x\right)+c_{4}\left(\psi_{8^{\text {th }} Y M}(x)-x\right)^{2}=f\left[\psi_{8^{\text {th }} Y M}(x), x, x\right]
\end{array}
$$

Solving Equation (14), we have

$$
\begin{aligned}
& c_{2}=\frac{\left(f\left[\psi_{2^{n d} N R}(x), x, x\right]\left(-S_{2}^{2} S_{3}+S_{2} S_{3}^{2}\right)+f\left[\psi_{4^{\text {th }} Y M}(x), x, x\right]\left(S_{1}^{2} S_{3}-S_{1} S_{3}^{2}\right)+f\left[\psi_{8^{\text {th }} Y M}(x), x, x\right]\left(-S_{1}^{2} S_{2}+S_{1} S_{2}^{2}\right)\right)}{-S_{1}^{2} S_{2}+S_{1} S_{2}^{2}+S_{1}^{2} S_{3}-S_{2}^{2} S_{3}-S_{1} S_{3}^{2}+S_{2} S_{3}^{2}}, \\
& c_{3}=\frac{\left(f\left[\psi_{2^{n d} N R}(x), x, x\right]\left(S_{2}^{2}-S_{3}^{2}\right)+f\left[\psi_{4^{\text {th }} Y M}(x), x, x\right]\left(-S_{1}^{2}+S_{3}^{2}\right)+f\left[\psi_{8^{\text {th }} Y M}(x), x, x\right]\left(S_{1}^{2}-S_{2}^{2}\right)\right)}{-S_{1}^{2} S_{2}+S_{1} S_{2}^{2}+S_{1}^{2} S_{3}-S_{2}^{2} S_{3}-S_{1} S_{3}^{2}+S_{2} S_{3}^{2}}, \\
& c_{4}=\frac{\left(f\left[\psi_{2^{n d} N R}(x), x, x\right]\left(-S_{2}+S_{3}\right)+f\left[\psi_{4^{\text {th }} Y M}(x), x, x\right]\left(S_{1}-S_{3}\right)+f\left[\psi_{8^{\text {th }} Y M}(x), x, x\right]\left(-S_{1}+S_{2}\right)\right)}{-S_{1}^{2} S_{2}+S_{1} S_{2}^{2}+S_{1}^{2} S_{3}-S_{2}^{2} S_{3}-S_{1} S_{3}^{2}+S_{2} S_{3}^{2}}, \\
& S_{1}=\psi_{2^{n d} N R}(x)-x, S_{2}=\psi_{4^{\text {th }} Y M}(x)-x, S_{3}=\psi_{8^{\text {th }} Y M}(x)-x .
\end{aligned}
$$

Further, using Equation (15), we have the estimation

$$
f^{\prime}\left(\psi_{8^{t h} Y M}(x)\right) \approx q^{\prime}\left(\psi_{8^{\text {th }} Y M}(x)\right)=c_{1}+2 c_{2}\left(\psi_{8^{\text {th }} Y M}(x)-x\right)+3 c_{3}\left(\psi_{8^{t h} Y M}(x)-x\right)^{2}+4 c_{4}\left(\psi_{8^{t h} Y M}(x)-x\right)^{3} .
$$

Finally, we propose a new optimal sixteenth order method as

$$
\psi_{16^{\text {th }}{ }_{M}}(x)=\psi_{8^{\text {th }} Y M}(x)-\frac{f\left(\psi_{8^{\text {th }} Y M}(x)\right)}{f^{\prime}(x)+2 c_{2}\left(\psi_{8^{\text {th }} Y M}(x)-x\right)+3 c_{3}\left(\psi_{8^{\text {th }} Y M}(x)-x\right)^{2}+4 c_{4}\left(\psi_{8^{t h} Y M}(x)-x\right)^{3}} .
$$

The efficiency of the method (16) is $E I_{16 t h Y M}=1.741$.

\section{Convergence Analysis}

In this section, we prove the convergence analysis of proposed IFs with help of Mathematica software.

Theorem 1. Let $f: D \subset \mathbb{R} \rightarrow \mathbb{R}$ be a sufficiently smooth function having continuous derivatives. If $f(x)$ has a simple root $x^{*}$ in the open interval $D$ and $x_{0}$ chosen in sufficiently small neighborhood of $x^{*}$, then the method $4^{\text {th }} Y M$ IFs (7) is of local fourth order convergence, and the $8^{\text {th }} Y M$ IFs (12) is of local eighth order convergence.

Proof. Let $e=x-x^{*}$ and $c[j]=\frac{f^{(j)}\left(x^{*}\right)}{j ! f^{\prime}\left(x^{*}\right)}, j=2,3,4, \ldots$. Expanding $f(x)$ and $f^{\prime}(x)$ about $x^{*}$ by Taylor's method, we have

$$
f(x)=f^{\prime}\left(x^{*}\right)\left(e+e^{2} c[2]+e^{3} c[3]+e^{4} c[4]+e^{5} c[5]+e^{6} c[6]+e^{7} c[7]+e^{8} c[8]+\ldots\right)
$$

and

$$
f^{\prime}(x)=f^{\prime}\left(x^{*}\right)\left(1+2 e c[2]+3 e^{2} c[3]+4 e^{3} c[4]+5 e^{4} c[5]+6 e^{5} c[6]+7 e^{6} c[7]+8 e^{7} c[8]+9 e^{8} c[9]+\ldots\right)
$$


Thus,

$$
\begin{aligned}
& \psi_{2^{n d} N R}(x)=x^{*}+c[2] e^{2}+\left(-2 c[2]^{2}+2 c[3]\right) e^{3}+\left(4 c[2]^{3}-7 c[2] c[3]+3 c[4]\right) e^{4}+\left(-8 c[2]^{4}\right. \\
& \left.+20 c[2]^{2} c[3]-6 c[3]^{2}-10 c[2] c[4]+4 c[5]\right) e^{5}+\left(16 c[2]^{5}-52 c[2]^{3} c[3]+28 c[2]^{2} c[4]-17 c[3] c[4]\right. \\
& \left.+c[2]\left(33 c[3]^{2}-13 c[5]\right)+5 c[6]\right) e^{6}-2\left(16 c[2]^{6}-64 c[2]^{4} c[3]-9 c[3]^{3}+36 c[2]^{3} c[4]+6 c[4]^{2}+9 c[2]^{2}\left(7 c[3]^{2}\right.\right. \\
& -2 c[5])+11 c[3] c[5]+c[2](-46 c[3] c[4]+8 c[6])-3 c[7]) e^{7}+\left(64 c[2]^{7}-304 c[2]^{5} c[3]\right. \\
& +176 c[2]^{4} c[4]+75 c[3]^{2} c[4]+c[2]^{3}\left(408 c[3]^{2}-92 c[5]\right)-31 c[4] c[5]-27 c[3] c[6] \\
& \left.+c[2]^{2}(-348 c[3] c[4]+44 c[6])+c[2]\left(-135 c[3]^{3}+64 c[4]^{2}+118 c[3] c[5]-19 c[7]\right)+7 c[8]\right) e^{8}+\ldots
\end{aligned}
$$

Expanding $f\left(\psi_{2^{n d} N R}(x)\right)$ about $x^{*}$ by Taylor's method, we have

$$
\begin{aligned}
& f\left(\psi_{2^{n d} N R}(x)\right)=f^{\prime}\left(x^{*}\right)\left(c[2] e^{2}+\left(-2 c[2]^{2}+2 c[3]\right) e^{3}+\left(5 c[2]^{3}-7 c[2] c[3]+3 c[4]\right) e^{4}-2\left(6 c[2]^{4}\right.\right. \\
& \left.-12 c[2]^{2} c[3]+3 c[3]^{2}+5 c[2] c[4]-2 c[5]\right) e^{5}+\left(28 c[2]^{5}-73 c[2]^{3} c[3]+34 c[2]^{2} c[4]-17 c[3] c[4]\right. \\
& \left.+c[2]\left(37 c[3]^{2}-13 c[5]\right)+5 c[6]\right) e^{6}-2\left(32 c[2]^{6}-103 c[2]^{4} c[3]-9 c[3]^{3}+52 c[2]^{3} c[4]+6 c[4]^{2}\right. \\
& \left.+c[2]^{2}\left(80 c[3]^{2}-22 c[5]\right)+11 c[3] c[5]+c[2](-52 c[3] c[4]+8 c[6])-3 c[7]\right) e^{7} \\
& +\left(144 c[2]^{7}-552 c[2]^{5} c[3]+297 c[2]^{4} c[4]+75 c[3]^{2} c[4]+2 c[2]^{3}\left(291 c[3]^{2}-67 c[5]\right)\right. \\
& -31 c[4] c[5]-27 c[3] c[6]+c[2]^{2}(-455 c[3] c[4]+54 c[6])+c[2]\left(-147 c[3]^{3}+73 c[4]^{2}\right. \\
& \left.+134 c[3] c[5]-19 c[7])+7 c[8]) e^{8}+\ldots\right)
\end{aligned}
$$

Using Equations (17)-(20) in divided difference techniques. We have

$$
\begin{aligned}
& f\left[\psi_{2^{n d} N R}(x), x, x\right]=f^{\prime}\left(x^{*}\right)\left(c[2]+2 c[3] e+(c[2] c[3]+3 c[4]) e^{2}+2\left(-c[2]^{2} c[3]+c[3]^{2}\right.\right. \\
& +c[2] c[4]+2 c[5]) e^{3}+\left(4 c[2]^{3} c[3]-3 c[2]^{2} c[4]+7 c[3] c[4]+c[2]\left(-7 c[3]^{2}+3 c[5]\right)+5 c[6]\right) e^{4} \\
& +\left(-8 c[2]^{4} c[3]-6 c[3]^{3}+4 c[2]^{3} c[4]+4 c[2]^{2}\left(5 c[3]^{2}-c[5]\right)+10 c[3] c[5]\right. \\
& \left.+4 c[2](-5 c[3] c[4]+c[6])+6\left(c[4]^{2}+c[7]\right)\right) e^{5}+\left(16 c[2]^{5} c[3]-4 c[2]^{4} c[4]\right. \\
& -25 c[3]^{2} c[4]+17 c[4] c[5]+c[2]^{3}\left(-52 c[3]^{2}+5 c[5]\right)+c[2]^{2}(46 c[3] c[4]-5 c[6]) \\
& \left.\left.+13 c[3] c[6]+c[2]\left(33 c[3]^{3}-14 c[4]^{2}-26 c[3] c[5]+5 c[7]\right)+7 c[8]\right) e^{6}+\ldots\right)
\end{aligned}
$$

Substituting Equations (18)-(21) into Equation (7), we obtain, after simplifications,

$$
\begin{aligned}
\psi_{4^{\text {th } Y M}}(x) & =x^{*}+\left(c[2]^{3}-c[2] c[3]\right) e^{4}-2\left(2 c[2]^{4}-4 c[2]^{2} c[3]+c[3]^{2}+c[2] c[4]\right) e^{5}+\left(10 c[2]^{5}-30 c[2]^{3} c[3]\right. \\
& \left.+12 c[2]^{2} c[4]-7 c[3] c[4]+3 c[2]\left(6 c[3]^{2}-c[5]\right)\right) e^{6}-2\left(10 c[2]^{6}-40 c[2]^{4} c[3]-6 c[3]^{3}\right. \\
& \left.+20 c[2]^{3} c[4]+3 c[4]^{2}+8 c[2]^{2}\left(5 c[3]^{2}-c[5]\right)+5 c[3] c[5]+c[2](-26 c[3] c[4]+2 c[6])\right) e^{7}+\left(36 c[2]^{7}\right. \\
& -178 c[2]^{5} c[3]+101 c[2]^{4} c[4]+50 c[3]^{2} c[4]+3 c[2]^{3}\left(84 c[3]^{2}-17 c[5]\right)-17 c[4] c[5]-13 c[3] c[6] \\
& \left.+c[2]^{2}(-209 c[3] c[4]+20 c[6])+c[2]\left(-91 c[3]^{3}+37 c[4]^{2}+68 c[3] c[5]-5 c[7]\right)\right) e^{8}+\ldots
\end{aligned}
$$


Expanding $f\left(\psi_{4^{\text {th }} Y M}(x)\right)$ about $x^{*}$ by Taylor's method, we have

$$
\begin{aligned}
f\left(\psi_{4^{t h} Y M}(x)\right) & =f^{\prime}\left(x^{*}\right)\left(\left(c[2]^{3}-c[2] c[3]\right) e^{4}-2\left(2 c[2]^{4}-4 c[2]^{2} c[3]+c[3]^{2}+c[2] c[4]\right) e^{5}+\left(10 c[2]^{5}\right.\right. \\
& \left.-30 c[2]^{3} c[3]+12 c[2]^{2} c[4]-7 c[3] c[4]+3 c[2]\left(6 c[3]^{2}-c[5]\right)\right) e^{6}-2\left(10 c[2]^{6}-40 c[2]^{4} c[3]\right. \\
& \left.-6 c[3]^{3}+20 c[2]^{3} c[4]+3 c[4]^{2}+8 c[2]^{2}\left(5 c[3]^{2}-c[5]\right)+5 c[3] c[5]+c[2](-26 c[3] c[4]+2 c[6])\right) e^{7} \\
& +\left(37 c[2]^{7}-180 c[2]^{5} c[3]+101 c[2]^{4} c[4]+50 c[3]^{2} c[4]+c[2]^{3}\left(253 c[3]^{2}-51 c[5]\right)-17 c[4] c[5]\right. \\
& \left.\left.-13 c[3] c[6]+c[2]^{2}(-209 c[3] c[4]+20 c[6])+c[2]\left(-91 c[3]^{3}+37 c[4]^{2}+68 c[3] c[5]-5 c[7]\right)\right) e^{8}+\ldots\right)
\end{aligned}
$$

Now,

$$
\begin{aligned}
f\left[\psi_{4^{\text {th }} Y M}(x), x, x\right] & =f^{\prime}\left(x^{*}\right)\left(c[2]+2 c[3] e+3 c[4] e^{2}+4 c[5] e^{3}+\left(c[2]^{3} c[3]-c[2] c[3]^{2}+5 c[6]\right) e^{4}\right. \\
& +\left(-4 c[2]^{4} c[3]+8 c[2]^{2} c[3]^{2}-2 c[3]^{3}+2 c[2]^{3} c[4]-4 c[2] c[3] c[4]+6 c[7]\right) e^{5} \\
& +\left(10 c[2]^{5} c[3]-8 c[2]^{4} c[4]+28 c[2]^{2} c[3] c[4]-11 c[3]^{2} c[4]+c[2]^{3}\left(-30 c[3]^{2}+3 c[5]\right)+\right. \\
& \left.\left.2 c[2]\left(9 c[3]^{3}-2 c[4]^{2}-3 c[3] c[5]\right)+7 c[8]\right) e^{6}+\ldots\right)
\end{aligned}
$$

Substituting Equations (19)-(21), (23) and (24) into Equation (12), we obtain, after simplifications,

$$
\psi_{8^{\text {th }} Y M}(x)-x^{*}=c[2]^{2}\left(c[2]^{2}-c[3]\right)\left(c[2]^{3}-c[2] c[3]+c[4]\right) e^{8}+O\left(e^{9}\right)
$$

Hence from Equations (22) and (25), we concluded that the convergence order of the $4^{\text {th }} Y M$ and $8^{\text {th }} Y M$ are four and eight respectively.

The following theorem is given without proof, which can be worked out with the help of Mathematica.

Theorem 2. Let $f: D \subset \mathbb{R} \rightarrow \mathbb{R}$ be a sufficiently smooth function having continuous derivatives. If $f(x)$ has a simple root $x^{*}$ in the open interval $D$ and $x_{0}$ chosen in sufficiently small neighborhood of $x^{*}$, then the method (16) is of local sixteenth order convergence and and it satisfies the error equation

$\psi_{16^{\text {th }} \Upsilon \mathrm{M}}(x)-x^{*}=\left(\left(c[2]^{4}\right)\left(\left(c[2]^{2}-c[3]\right)^{2}\right)\left(c[2]^{3}-c[2] c[3]+c[4]\right)\left(c[2]^{4}-c[2]^{2} c[3]+c[2] c[4]-c[5]\right)\right) e^{16}+O\left(e^{17}\right)$.

\section{Numerical Examples}

In this section, numerical results on some test functions are compared for the new methods $4^{\text {th }} Y M, 8^{\text {th }} Y M$ and $16^{\text {th }} Y M$ with some existing eighth order methods and Newton's method. Numerical computations have been carried out in the MATLAB software with 500 significant digits. We have used the stopping criteria for the iterative process satisfying error $=\left|x_{N}-x_{N-1}\right|<\epsilon$, where $\epsilon=10^{-50}$ and $N$ is the number of iterations required for convergence. The computational order of convergence is given by ([17])

$$
\rho=\frac{\ln \left|\left(x_{N}-x_{N-1}\right) /\left(x_{N-1}-x_{N-2}\right)\right|}{\ln \left|\left(x_{N-1}-x_{N-2}\right) /\left(x_{N-2}-x_{N-3}\right)\right|} .
$$

We consider the following iterative methods for solving nonlinear equations for the purpose of comparison: $\psi_{4^{\text {th }} S B}$, a method proposed by Sharma et al. [18]:

$$
y=x-\frac{2 f(x)}{3 f^{\prime}(x)}, \psi_{4^{\text {th }}}(x)=x-\left(-\frac{1}{2}+\frac{9}{8} \frac{f^{\prime}(x)}{f^{\prime}(y)}+\frac{3}{8} \frac{f^{\prime}(y)}{f^{\prime}(x)}\right) \frac{f(x)}{f^{\prime}(x)} .
$$

$\psi_{4^{\text {th }} \mathrm{CLND}}$, a method proposed by Chun et al. [19]:

$$
y=x-\frac{2 f(x)}{3 f^{\prime}(x)}, \psi_{4^{\text {th }} C L N D}(x)=x-\frac{16 f(x) f^{\prime}(x)}{-5 f^{\prime}(x)^{2}+30 f^{\prime}(x) f^{\prime}(y)-9 f^{\prime}(y)^{2}} .
$$


$\psi_{4^{\text {th }} \text { S }}$, a method proposed by Singh et al. [20]:

$$
y=x-\frac{2}{3} \frac{f(x)}{f^{\prime}(x)}, \psi_{4^{t h} S J}(x)=x-\left(\frac{17}{8}-\frac{9}{4} \frac{f^{\prime}(y)}{f^{\prime}(x)}+\frac{9}{8}\left(\frac{f^{\prime}(y)}{f^{\prime}(x)}\right)^{2}\right)\left(\frac{7}{4}-\frac{3}{4} \frac{f^{\prime}(y)}{f^{\prime}\left(x_{n}\right)}\right) \frac{f(x)}{f^{\prime}(x)} .
$$

$\psi_{8^{\text {th }} K T}$, a method proposed by Kung-Traub [2]:

$$
\begin{aligned}
& y=x-\frac{f(x)}{f^{\prime}(x)}, z=y-\frac{f(y) * f(x)}{(f(x)-f(y))^{2}} \frac{f(x)}{f^{\prime}(x)} \\
& \psi_{8^{\text {th } K T}}(x)=z-\frac{f(x)}{f^{\prime}(x)} \frac{f(x) f(y) f(z)}{(f(x)-f(y))^{2}} \frac{f(x)^{2}+f(y)(f(y)-f(z))}{(f(x)-f(z))^{2}(f(y)-f(z))} .
\end{aligned}
$$

$\psi_{8^{t h} L W}$, a method proposed by Liu et al. [8]

$$
\begin{aligned}
& y=x-\frac{f(x)}{f^{\prime}(x)}, z=y-\frac{f(x)}{f(x)-2 f(y)} \frac{f(y)}{f^{\prime}(x)} \\
& \psi_{8^{\text {th }} L W}(x)=z-\frac{f(z)}{f^{\prime}(x)}\left(\left(\frac{f(x)-f(y)}{f(x)-2 f(y)}\right)^{2}+\frac{f(z)}{f(y)-f(z)}+\frac{4 f(z)}{f(x)+f(z)}\right) .
\end{aligned}
$$

$\psi_{8^{t h} P N P D^{\prime}}$ a method proposed by Petkovic et al. [11]

$$
\begin{aligned}
& y=x-\frac{f(x)}{f^{\prime}(x)}, z=x-\left(\left(\frac{f(y)}{f(x)}\right)^{2}-\frac{f(x)}{f(y)-f(x)}\right) \frac{f(x)}{f^{\prime}(x)}, \psi_{8^{\text {th }} P N P D}(x)=z-\frac{f(z)}{f^{\prime}(x)}\left(\varphi(t)+\frac{f(z)}{f(y)-f(z)}+\frac{4 f(z)}{f(x)}\right), \\
& \text { where } \varphi(t)=1+2 t+2 t^{2}-t^{3} \text { and } t=\frac{f(y)}{f(x)} .
\end{aligned}
$$

$\psi_{8^{\text {th }}}$ A1 , a method proposed by Sharma et al. [12]

$$
y=x-\frac{f(x)}{f^{\prime}(x)}, z=y-\left(3-2 \frac{f[y, x]}{f^{\prime}(x)}\right) \frac{f(y)}{f^{\prime}(x)}, \psi_{8^{\text {th }} S A 1}(x)=z-\frac{f(z)}{f^{\prime}(x)}\left(\frac{f^{\prime}(x)-f[y, x]+f[z, y]}{2 f[z, y]-f[z, x]}\right) .
$$

$\psi_{8^{\text {th }} S A 2}$, a method proposed by Sharma et al. [13]

$$
y=x-\frac{f(x)}{f^{\prime}(x)}, z=y-\frac{f(y)}{2 f[y, x]-f^{\prime}(x)}, \psi_{8^{\text {th }} S A 2}(x)=z-\frac{f[z, y]}{f[z, x]} \frac{f(z)}{2 f[z, y]-f[z, x]}
$$

$\psi_{8^{t h} C F G T}$, a method proposed by Cordero et al. [6]

$$
\begin{array}{r}
y=x-\frac{f(x)}{f^{\prime}(x)}, z=y-\frac{f(y)}{f^{\prime}(x)} \frac{1}{1-2 t+t^{2}-t^{3} / 2}, \psi_{8^{\text {th }} \text { CFGT }}(x)= \\
z-\frac{1+3 r}{1+r} \frac{f(z)}{f[z, y]+f[z, x, x](z-y)}, r=\frac{f(z)}{f(x)} .
\end{array}
$$

$\psi_{8^{\text {th }} \mathrm{CTV}}$, a method proposed by Cordero et al. [7]

$$
y=x-\frac{f(x)}{f^{\prime}(x)}, z=x-\frac{1-t}{1-2 t} \frac{f(x)}{f^{\prime}(x)}, \psi_{8^{t h} C T V}(x)=z-\left(\frac{1-t}{1-2 t}-v\right)^{2} \frac{1}{1-3 v} \frac{f(z)}{f^{\prime}(x)}, v=\frac{f(z)}{f(y)} .
$$

Table 1 shows the efficiency indices of the new methods with some known methods. 
Table 1. Comparison of Efficiency Indices.

\begin{tabular}{cccc}
\hline Methods & $p$ & $d$ & $\boldsymbol{E I}$ \\
\hline $2^{\text {nd }} \mathrm{NR}$ & 2 & 2 & 1.414 \\
$4^{\text {th }} \mathrm{SB}$ & 4 & 3 & 1.587 \\
$4^{\text {th }} \mathrm{CLND}$ & 4 & 3 & 1.587 \\
$4^{\text {th }} \mathrm{SJ}$ & 4 & 3 & 1.587 \\
$4^{\text {th }} \mathrm{YM}$ & 4 & 3 & 1.587 \\
$8^{\text {th }} \mathrm{KT}$ & 8 & 4 & 1.682 \\
$8^{\text {th }} \mathrm{LW}$ & 8 & 4 & 1.682 \\
$8^{\text {th }} \mathrm{PNPD}$ & 8 & 4 & 1.682 \\
$8^{\text {th }} \mathrm{SA} 1$ & 8 & 4 & 1.682 \\
$8^{\text {th }} \mathrm{SA} 2$ & 8 & 4 & 1.682 \\
$8^{\text {th }} \mathrm{CFGT}$ & 8 & 4 & 1.682 \\
$8^{\text {th }} \mathrm{CTV}$ & 8 & 4 & 1.682 \\
$8^{\text {th }} \mathrm{YM}$ & 8 & 4 & 1.682 \\
$16^{\text {th }} \mathrm{YM}$ & 16 & 5 & 1.741 \\
\hline
\end{tabular}

The following test functions and their simple zeros for our study are given below [10]:

$$
\begin{aligned}
f_{1}(x) & =\sin (2 \cos x)-1-x^{2}+e^{\sin \left(x^{3}\right)}, & x^{*} & =-0.7848959876612125352 \ldots \\
f_{2}(x) & =x e^{x^{2}}-\sin ^{2} x+3 \cos x+5, & x^{*} & =-1.2076478271309189270 \ldots \\
f_{3}(x) & =x^{3}+4 x^{2}-10, & x^{*} & =1.3652300134140968457 \ldots \\
f_{4}(x) & =\sin (x)+\cos (x)+x, & x^{*} & =-0.4566247045676308244 \ldots \\
f_{5}(x) & =\frac{x}{2}-\sin x, & x^{*} & =1.8954942670339809471 \ldots \\
f_{6}(x) & =x^{2}+\sin \left(\frac{x}{5}\right)-\frac{1}{4}, & x^{*} & =0.4099920179891371316 \ldots
\end{aligned}
$$

Table 2, shows that corresponding results for $f_{1}-f_{6}$. We observe that proposed method $4^{\text {th }} Y M$ is converge in a lesser or equal number of iterations and with least error when compare to compared methods. Note that $4^{\text {th }} S B$ and $4^{\text {th }} S J$ methods are getting diverge in $f_{5}$ function. Hence, the proposed method $4^{\text {th }} Y M$ can be considered competent enough to existing other compared equivalent methods.

Also, from Tables $3-5$ are shows the corresponding results for $f_{1}-f_{6}$. The computational order of convergence agrees with the theoretical order of convergence in all the functions. Note that $8^{\text {th }} P N P D$ method is getting diverge in $f_{5}$ function and all other compared methods are converges with least error. Also, function $f_{1}$ having least error in $8^{\text {th }} C F G T$, function $f_{2}$ having least error in $8^{\text {th }} C T V$, functions $f_{3}$ and $f_{4}$ having least error in $8^{\text {th }} Y M$, function $f_{5}$ having least error in $8^{\text {th }} S A 2$, function $f_{6}$ having least error in $8^{\text {th }} C F G T$. The proposed $16^{\text {th }} Y M$ method converges less number of iteration with least error in all the tested functions. Hence, the $16^{\text {th }} Y M$ can be considered competent enough to existing other compared equivalent methods. 
Table 2. Numerical results for nonlinear equations.

\begin{tabular}{|c|c|c|c|c|c|c|c|c|}
\hline \multirow[t]{2}{*}{ Methods } & \multicolumn{4}{|c|}{$f_{1}(x), x_{0}=-0.9$} & \multicolumn{4}{|c|}{$f_{2}(x), x_{0}=-1.6$} \\
\hline & $N$ & $\left|x_{1}-x_{0}\right|$ & $\left|x_{N}-x_{N-1}\right|$ & $\rho$ & $N$ & $\left|x_{1}-x_{0}\right|$ & $\left|x_{N}-x_{N-1}\right|$ & $\rho$ \\
\hline $2^{\text {nd }} N R(4)$ & 7 & 0.1080 & $7.7326 \times 10^{-74}$ & 1.99 & 9 & 0.2044 & $9.2727 \times 10^{-74}$ & 1.99 \\
\hline $4^{\text {th }} S B(26)$ & 4 & 0.1150 & $9.7275 \times 10^{-64}$ & 3.99 & 5 & 0.3343 & $1.4237 \times 10^{-65}$ & 3.99 \\
\hline $4^{\text {th }} C L N D$ (27) & 4 & 0.1150 & $1.4296 \times 10^{-64}$ & 3.99 & 5 & 0.3801 & $1.1080 \times 10^{-72}$ & 3.99 \\
\hline $4^{\text {th }} S J(28)$ & 4 & 0.1150 & $3.0653 \times 10^{-62}$ & 3.99 & 5 & 0.3190 & $9.9781 \times 10^{-56}$ & 3.99 \\
\hline $4^{\text {th }} Y M(7)$ & 4 & 0.1150 & $6.0046 \times 10^{-67}$ & 3.99 & 5 & 0.3737 & $7.2910 \times 10^{-120}$ & 4.00 \\
\hline Methods & \multicolumn{4}{|c|}{$f_{3}(x), x_{0}=0.9$} & \multicolumn{4}{|c|}{$f_{4}(x), x_{0}=-1.9$} \\
\hline $2^{n d} N R(4)$ & 8 & 0.6263 & $1.3514 \times 10^{-72}$ & 2.00 & 8 & 1.9529 & $1.6092 \times 10^{-72}$ & 1.99 \\
\hline $4^{\text {th }} S B(26)$ & 5 & 0.5018 & $4.5722 \times 10^{-106}$ & 3.99 & 5 & 1.5940 & $6.0381 \times 10^{-92}$ & 3.99 \\
\hline $4^{\text {th }} C L N D$ (27) & 5 & 0.5012 & $4.7331 \times 10^{-108}$ & 3.99 & 5 & 1.5894 & $2.7352 \times 10^{-93}$ & 3.99 \\
\hline $4^{\text {th }} S J(28)$ & 5 & 0.4767 & $3.0351 \times 10^{-135}$ & 3.99 & 5 & 1.5776 & $9.5025 \times 10^{-95}$ & 3.99 \\
\hline $4^{\text {th }} Y M(7)$ & 5 & 0.4735 & $2.6396 \times 10^{-156}$ & 3.99 & 5 & 1.5519 & $1.4400 \times 10^{-102}$ & 3.99 \\
\hline Methods & \multicolumn{4}{|c|}{$f_{5}(x), x_{0}=1.2$} & \multicolumn{4}{|c|}{$f_{6}(x), x_{0}=0.8$} \\
\hline $2^{n d} N R(4)$ & 9 & 2.4123 & $1.3564 \times 10^{-83}$ & 1.99 & 8 & 0.3056 & $3.2094 \times 10^{-72}$ & 1.99 \\
\hline $4^{\text {th }} S B(26)$ & & & Diverge & & 5 & 0.3801 & $2.8269 \times 10^{-122}$ & 3.99 \\
\hline $4^{\text {th }} C L N D(27)$ & 14 & 0.0566 & $6.8760 \times 10^{-134}$ & 3.99 & 5 & 0.3812 & $7.8638 \times 10^{-127}$ & 3.99 \\
\hline $4^{\text {th }} S J(28)$ & & & Diverge & & 5 & 0.3780 & $1.4355 \times 10^{-114}$ & 3.99 \\
\hline $4^{\text {th }} Y M(7)$ & 6 & 1.2887 & $2.3155 \times 10^{-149}$ & 3.99 & 5 & 0.3840 & $1.1319 \times 10^{-143}$ & 3.99 \\
\hline
\end{tabular}

Table 3. Numerical results for nonlinear equations.

\begin{tabular}{|c|c|c|c|c|c|c|c|c|}
\hline \multirow[t]{2}{*}{ Methods } & \multicolumn{4}{|c|}{$f_{1}(x), x_{0}=-0.9$} & \multicolumn{4}{|c|}{$f_{2}(x), x_{0}=-1.6$} \\
\hline & $N$ & $\left|x_{1}-x_{0}\right|$ & $\left|x_{N}-x_{N-1}\right|$ & $\rho$ & $N$ & $\left|x_{1}-x_{0}\right|$ & $\left|x_{N}-x_{N-1}\right|$ & $\rho$ \\
\hline $8^{\text {th }} K T$ (29) & 3 & 0.1151 & $1.6238 \times 10^{-61}$ & 7.91 & 4 & 0.3876 & $7.2890 \times 10^{-137}$ & 7.99 \\
\hline $8^{\text {th }} L W(30)$ & 3 & 0.1151 & $4.5242 \times 10^{-59}$ & 7.91 & 4 & 0.3904 & $1.1195 \times 10^{-170}$ & 8.00 \\
\hline $8^{\text {th }} P N P D(31)$ & 3 & 0.1151 & $8.8549 \times 10^{-56}$ & 7.87 & 4 & 0.3734 & $2.3461 \times 10^{-85}$ & 7.99 \\
\hline $8^{\text {th }} S A 1$ (32) & 3 & 0.1151 & $3.4432 \times 10^{-60}$ & 7.88 & 4 & 0.3983 & $8.4343 \times 10^{-121}$ & 8.00 \\
\hline $8^{\text {th }} S A 2$ (33) & 3 & 0.1151 & $6.9371 \times 10^{-67}$ & 7.99 & 4 & 0.3927 & $5.9247 \times 10^{-225}$ & 7.99 \\
\hline $8^{\text {th }}$ CFGT (34) & 3 & 0.1151 & $1.1715 \times 10^{-82}$ & 7.77 & 5 & 0.1532 & $2.0650 \times 10^{-183}$ & 7.99 \\
\hline $8^{\text {th }} \mathrm{CTV}$ (35) & 3 & 0.1151 & $4.4923 \times 10^{-61}$ & 7.94 & 4 & 0.3925 & $2.3865 \times 10^{-252}$ & 7.99 \\
\hline $8^{\text {th }} Y M(12)$ & 3 & 0.1151 & $1.1416 \times 10^{-70}$ & 7.96 & 4 & 0.3896 & $8.9301 \times 10^{-163}$ & 8.00 \\
\hline $16^{\text {th }} Y M(16)$ & 3 & 0.1151 & 0 & 15.99 & 3 & 0.3923 & $3.5535 \times 10^{-85}$ & 16.20 \\
\hline
\end{tabular}

Table 4. Numerical results for nonlinear equations.

\begin{tabular}{|c|c|c|c|c|c|c|c|c|}
\hline \multirow[t]{2}{*}{ Methods } & \multicolumn{4}{|c|}{$f_{3}(x), x_{0}=0.9$} & \multicolumn{4}{|c|}{$f_{4}(x), x_{0}=-1.9$} \\
\hline & $N$ & $\left|x_{1}-x_{0}\right|$ & $\left|x_{N}-x_{N-1}\right|$ & $\rho$ & $N$ & $\left|x_{1}-x_{0}\right|$ & $\left|x_{N}-x_{N-1}\right|$ & $\rho$ \\
\hline $8^{\text {th }} K T$ (29) & 4 & 0.4659 & $5.0765 \times 10^{-216}$ & 7.99 & 4 & 1.4461 & $5.5095 \times 10^{-204}$ & 8.00 \\
\hline $8^{t h} L W(30)$ & 4 & 0.4660 & $2.7346 \times 10^{-213}$ & 7.99 & 4 & 1.4620 & $3.7210 \times 10^{-146}$ & 8.00 \\
\hline $8^{\text {th }}$ PNPD (31) & 4 & 0.3821 & $9.9119 \times 10^{-71}$ & 8.02 & 4 & 1.3858 & $2.0603 \times 10^{-116}$ & 7.98 \\
\hline $8^{\text {th }} S A 1$ (32) & 4 & 0.4492 & $1.5396 \times 10^{-122}$ & 8.00 & 4 & 1.4170 & $2.2735 \times 10^{-136}$ & 7.99 \\
\hline $8^{\text {th }} S A 2$ (33) & 4 & 0.4652 & $4.1445 \times 10^{-254}$ & 7.98 & 4 & 1.4339 & $2.5430 \times 10^{-166}$ & 7.99 \\
\hline $8^{\text {th }}$ CFGT (34) & 4 & 0.4654 & $2.4091 \times 10^{-260}$ & 7.99 & 4 & 1.4417 & $4.7007 \times 10^{-224}$ & 7.99 \\
\hline $8^{\text {th }} C T V$ (35) & 4 & 0.4652 & $3.8782 \times 10^{-288}$ & 8.00 & 4 & 1.3957 & $3.7790 \times 10^{-117}$ & 7.99 \\
\hline $8^{\text {th }} Y M(12)$ & 4 & 0.4653 & $3.5460 \times 10^{-309}$ & 7.99 & 4 & 1.4417 & $2.9317 \times 10^{-229}$ & 7.99 \\
\hline $16^{\text {th }} Y M(16)$ & 3 & 0.4652 & $3.6310 \times 10^{-154}$ & 16.13 & 3 & 1.4434 & $1.8489 \times 10^{-110}$ & 16.36 \\
\hline
\end{tabular}


Table 5. Numerical results for nonlinear equations.

\begin{tabular}{ccccccccc}
\hline Methods & \multicolumn{3}{c}{$f_{\mathbf{5}}(\boldsymbol{x}), \boldsymbol{x}_{\mathbf{0}}=\mathbf{1 . 2}$} & \multicolumn{4}{c}{$f_{\mathbf{6}}(x), x_{\mathbf{0}}=\mathbf{0 . 8}$} \\
\hline & $N$ & $\left|x_{\mathbf{1}}-x_{\mathbf{0}}\right|$ & $\left|x_{N}-x_{N-\mathbf{1}}\right|$ & $\rho$ & $N$ & $\left|x_{\mathbf{1}}-x_{\mathbf{0}}\right|$ & $\left|x_{N}-x_{N-1}\right|$ & $\rho$ \\
\hline $8^{\text {th }}$ KT (29) & 5 & 1.8787 & $2.6836 \times 10^{-182}$ & 7.99 & 4 & 0.3898 & $6.0701 \times 10^{-234}$ & 7.99 \\
$8^{\text {th }}$ LW (30) & 6 & 40.5156 & $4.6640 \times 10^{-161}$ & 7.99 & 4 & 0.3898 & $6.1410 \times 10^{-228}$ & 7.99 \\
$8^{\text {th }}$ PNPD (31) & & & Diverge & & 4 & 0.3894 & $3.6051 \times 10^{-190}$ & 7.99 \\
$8^{\text {th }}$ SA1 (32) & 7 & 891.9802 & $2.1076 \times 10^{-215}$ & 9.00 & 4 & 0.3901 & $5.9608 \times 10^{-245}$ & 8.00 \\
$8^{\text {th }}$ SA2 (33) & 4 & 0.7161 & $5.3670 \times 10^{-128}$ & 7.99 & 4 & 0.3900 & $8.3398 \times 10^{-251}$ & 8.61 \\
$8^{\text {th }}$ CFGT (34) & 5 & 2.8541 & 0 & 7.99 & 4 & 0.3900 & 0 & 7.99 \\
$8^{\text {th }}$ CTV (35) & 5 & 0.6192 & $1.6474 \times 10^{-219}$ & 9.00 & 4 & 0.3901 & $1.0314 \times 10^{-274}$ & 8.00 \\
$8^{\text {th }}$ YM (12) & 4 & 0.7733 & $1.3183 \times 10^{-87}$ & 7.98 & 4 & 0.3900 & $1.2160 \times 10^{-286}$ & 7.99 \\
$16^{\text {th } Y M(16)}$ & 4 & 0.6985 & 0 & 16.10 & 3 & 0.3900 & $1.1066 \times 10^{-143}$ & 15.73 \\
\hline
\end{tabular}

\section{Applications to Some Real World Problems}

\subsection{Projectile Motion Problem}

We consider the classical projectile problem $[21,22]$ in which a projectile is launched from a tower of height $h>0$, with initial speed $v$ and at an angle $\theta$ with respect to the horizontal onto a hill, which is defined by the function $\omega$, called the impact function which is dependent on the horizontal distance, $x$. We wish to find the optimal launch angle $\theta_{m}$ which maximizes the horizontal distance. In our calculations, we neglect air resistances.

The path function $y=P(x)$ that describes the motion of the projectile is given by

$$
P(x)=h+x \tan \theta-\frac{g x^{2}}{2 v^{2}} \sec ^{2} \theta
$$

When the projectile hits the hill, there is a value of $x$ for which $P(x)=\omega(x)$ for each value of $x$. We wish to find the value of $\theta$ that maximize $x$.

$$
\omega(x)=P(x)=h+x \tan \theta-\frac{g x^{2}}{2 v^{2}} \sec ^{2} \theta
$$

Differentiating Equation (37) implicitly w.r.t. $\theta$, we have

$$
\omega^{\prime}(x) \frac{d x}{d \theta}=x \sec ^{2} \theta+\frac{d x}{d \theta} \tan \theta-\frac{g}{v^{2}}\left(x^{2} \sec ^{2} \theta \tan \theta+x \frac{d x}{d \theta} \sec ^{2} \theta\right)
$$

Setting $\frac{d x}{d \theta}=0$ in Equation (38), we have

$$
x_{m}=\frac{v^{2}}{g} \cot \theta_{m}
$$

or

$$
\theta_{m}=\arctan \left(\frac{v^{2}}{g x_{m}}\right)
$$

An enveloping parabola is a path that encloses and intersects all possible paths. Henelsmith [23] derived an enveloping parabola by maximizing the height of the projectile fora given horizontal distance $x$, which will give the path that encloses all possible paths. Let $w=\tan \theta$, then Equation (36) becomes

$$
y=P(x)=h+x w-\frac{g x^{2}}{2 v^{2}}\left(1+w^{2}\right)
$$


Differentiating Equation (41) w.r.t. $w$ and setting $y^{\prime}=0$, Henelsmith obtained

$$
\begin{aligned}
& y^{\prime}=x-\frac{x g^{2}}{v^{2}}(w)=0 \\
& w=\frac{v^{2}}{g x}
\end{aligned}
$$

so that the enveloping parabola defined by

$$
y_{m}=\rho(x)=h+\frac{v^{2}}{2 g}-\frac{g x^{2}}{2 v^{2}}
$$

The solution to the projectile problem requires first finding $x_{m}$ which satisfies $\rho(x)=\omega(x)$ and solving for $\theta_{m}$ in Equation (40) because we want to find the point at which the enveloping parabola $\rho$ intersects the impact function $\omega$, and then find $\theta$ that corresponds to this point on the enveloping parabola. We choose a linear impact function $\omega(x)=0.4 x$ with $h=10$ and $v=20$. We let $g=9.8$. Then we apply our IFs starting from $x_{0}=30$ to solve the non-linear equation

$$
f(x)=\rho(x)-\omega(x)=h+\frac{v^{2}}{2 g}-\frac{g x^{2}}{2 v^{2}}-0.4 x
$$

whose root is given by $x_{m}=36.102990117 \ldots . .$. and

$$
\theta_{m}=\arctan \left(\frac{v^{2}}{g x_{m}}\right)=48.5^{\circ} .
$$

Figure 1 shows the intersection of the path function, the enveloping parabola and the linear impact function for this application. The approximate solutions are calculated correct to 500 significant figures. The stopping criterion $\left|x_{N}-x_{N-1}\right|<\epsilon$, where $\epsilon=10^{-50}$ is used. Table 6 shows that proposed method $16^{\text {th }} \mathrm{YM}$ is converging better than other compared methods. Also, we observe that the computational order of convergence agrees with the theoretical order of convergence.

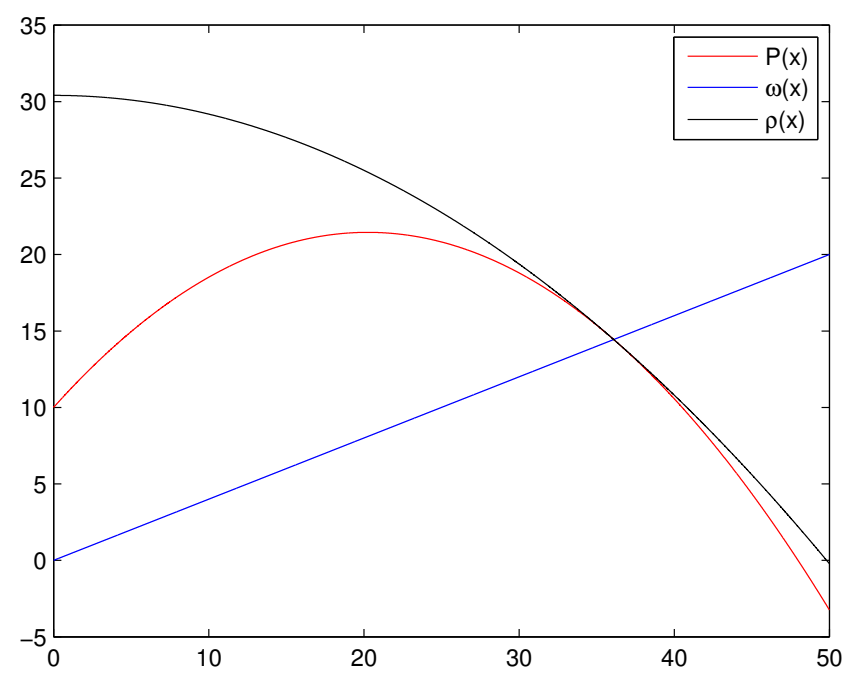

Figure 1. The enveloping parabola with linear impact function. 
Table 6. Results of projectile problem.

\begin{tabular}{ccccc}
\hline IF & $N$ & Error & cpu Time $(s)$ & $\rho$ \\
\hline $2^{\text {nd }} \mathrm{NR}$ & 7 & $4.3980 \times 10^{-76}$ & 1.074036 & 1.99 \\
$4^{\text {th }} \mathrm{YM}$ & 4 & $4.3980 \times 10^{-76}$ & 0.902015 & 3.99 \\
$8^{\text {th }} \mathrm{KT}$ & 3 & $1.5610 \times 10^{-66}$ & 0.658235 & 8.03 \\
$8^{\text {th }} \mathrm{LW}$ & 3 & $7.8416 \times 10^{-66}$ & 0.672524 & 8.03 \\
$8^{\text {th }} \mathrm{PNPD}$ & 3 & $4.2702 \times 10^{-57}$ & 0.672042 & 8.05 \\
$8^{\text {th }} \mathrm{SA} 1$ & 3 & $1.2092 \times 10^{-61}$ & 0.654623 & 8.06 \\
$8^{\text {th }} \mathrm{CTV}$ & 3 & $3.5871 \times 10^{-73}$ & 0.689627 & 8.02 \\
$8^{\text {th }} \mathrm{YM}$ & 3 & $4.3980 \times 10^{-76}$ & 0.618145 & 8.02 \\
$16^{\text {th }} \mathrm{YM}$ & 3 & 0 & 0.512152 & 16.01 \\
\hline
\end{tabular}

\subsection{Planck's Radiation Law Problem}

We consider the following Planck's radiation law problem found in [24]:

$$
\varphi(\lambda)=\frac{8 \pi \operatorname{ch} \lambda^{-5}}{e^{c h / \lambda k T}-1}
$$

which calculates the energy density within an isothermal blackbody. Here, $\lambda$ is the wavelength of the radiation, $T$ is the absolute temperature of the blackbody, $k$ is Boltzmann's constant, $h$ is the Planck's constant and $c$ is the speed of light. Suppose, we would like to determine wavelength $\lambda$ which corresponds to maximum energy density $\varphi(\lambda)$. From (44), we get

$$
\varphi^{\prime}(\lambda)=\left(\frac{8 \pi c h \lambda^{-6}}{e^{c h / \lambda k T}-1}\right)\left(\frac{(\operatorname{ch} / \lambda k T) e^{c h / \lambda k T}}{e^{c h / \lambda k T}-1}-5\right)=A \cdot B
$$

It can be checked that a maxima for $\varphi$ occurs when $B=0$, that is, when

$$
\left(\frac{(c h / \lambda k T) e^{c h / \lambda k T}}{e^{c h / \lambda k T}-1}\right)=5 \text {. }
$$

Here putting $x=c h / \lambda k T$, the above equation becomes

$$
1-\frac{x}{5}=e^{-x}
$$

Define

$$
f(x)=e^{-x}-1+\frac{x}{5} .
$$

The aim is to find a root of the equation $f(x)=0$. Obviously, one of the root $x=0$ is not taken for discussion. As argued in [24], the left-hand side of (45) is zero for $x=5$ and $e^{-5} \approx 6.74 \times 10^{-3}$. Hence, it is expected that another root of the equation $f(x)=0$ might occur near $x=5$. The approximate root of the Equation (46) is given by $x^{*} \approx 4.96511423174427630369$ with $x_{0}=3$. Consequently, the wavelength of radiation $(\lambda)$ corresponding to which the energy density is maximum is approximated as

$$
\lambda \approx \frac{c h}{(k T) 4.96511423174427630369} .
$$

Table 7 shows that proposed method $16^{\text {th }} \mathrm{YM}$ is converging better than other compared methods. Also, we observe that the computational order of convergence agrees with the theoretical order of convergence. 
Table 7. Results of Planck's radiation law problem.

\begin{tabular}{ccccc}
\hline IF & $N$ & Error & cpu Time $(s)$ & $\rho$ \\
\hline $2^{\text {nd }} \mathrm{NR}$ & 7 & $1.8205 \times 10^{-70}$ & 0.991020 & 2.00 \\
$4^{\text {th }} \mathrm{YM}$ & 5 & $1.4688 \times 10^{-181}$ & 0.842220 & 4.00 \\
$8^{\text {th }} \mathrm{KT}$ & 4 & $4.0810 \times 10^{-288}$ & 0.808787 & 7.99 \\
$8^{\text {th }} \mathrm{LW}$ & 4 & $3.1188 \times 10^{-268}$ & 0.801304 & 7.99 \\
$8^{\text {th }} \mathrm{PNPD}$ & 4 & $8.0615 \times 10^{-260}$ & 0.800895 & 7.99 \\
$8^{\text {th }} \mathrm{SA} 1$ & 4 & $1.9335 \times 10^{-298}$ & 0.791706 & 8.00 \\
$8^{\text {th }} \mathrm{CTV}$ & 4 & $5.8673 \times 10^{-282}$ & 0.831006 & 8.00 \\
$8^{\text {th }} \mathrm{YM}$ & 4 & $2.5197 \times 10^{-322}$ & 0.855137 & 8.00 \\
$16^{\text {th }} \mathrm{YM}$ & 3 & $8.3176 \times 10^{-153}$ & 0.828053 & 16.52 \\
\hline
\end{tabular}

Hereafter, we will study the optimal fourth and eighth order methods along with Newton's method.

\section{Corresponding Conjugacy Maps for Quadratic Polynomials}

In this section, we discuss the rational map $R_{p}$ arising from $2^{\text {nd }} N R$, proposed methods $4^{\text {th }} Y M$ and $8^{\text {th }} Y M$ applied to a generic polynomial with simple roots.

Theorem 3. $\left(2^{\text {nd }} N R\right)$ [18] For a rational map $R_{p}(z)$ arising from Newton's method (4) applied to $p(z)=$ $(z-a)(z-b), a \neq b, R_{p}(z)$ is conjugate via the a Möbius transformation given by $M(z)=(z-a) /(z-b)$ to

$$
S(z)=z^{2}
$$

Theorem 4. (4 $\left.4^{\text {th }} Y M\right)$ For a rational map $R_{p}(z)$ arising from Proposed Method (7) applied to $p(z)=(z-$ $a)(z-b), a \neq b, R_{p}(z)$ is conjugate via the a Möbius transformation given by $M(z)=(z-a) /(z-b)$ to

$$
S(z)=z^{4}
$$

Proof. Let $p(z)=(z-a)(z-b), a \neq b$, and let $M$ be Möbius transformation given by $M(z)=$ $(z-a) /(z-b)$ with its inverse $M^{-1}(z)=\frac{(z b-a)}{(z-1)}$, which may be considered as map from $\mathbb{C} \cup\{\infty\}$. We then have

$$
S(z)=M \circ R_{p} \circ M^{-1}(z)=M\left(R_{p}\left(\frac{z b-a}{z-1}\right)\right)=z^{4} .
$$

Theorem 5. ( $\left.8^{\text {th }} Y M\right)$ For a rational map $R_{p}(z)$ arising from Proposed Method (12) applied to $p(z)=(z-$ $a)(z-b), a \neq b, R_{p}(z)$ is conjugate via the a Möbius transformation given by $M(z)=(z-a) /(z-b)$ to

$$
S(z)=z^{8}
$$

Proof. Let $p(z)=(z-a)(z-b), a \neq b$, and let $M$ be Möbius transformation given by $M(z)=$ $(z-a) /(z-b)$ with its inverse $M^{-1}(z)=\frac{(z b-a)}{(z-1)}$, which may be considered as map from $\mathbb{C} \cup\{\infty\}$. We then have

$$
S(z)=M \circ R_{p} \circ M^{-1}(z)=M\left(R_{p}\left(\frac{z b-a}{z-1}\right)\right)=z^{8} .
$$

Remark 1. The methods (29)-(35) are given without proof, which can be worked out with the help of Mathematica.

Remark 2. All the maps obtained above are of the form $S(z)=z^{p} R(z)$, where $R(z)$ is either unity or a rational function and $p$ is the order of the method. 


\section{Basins of Attraction}

The study of dynamical behavior of the rational function associated to an iterative method gives important information about convergence and stability of the method. The basic definitions and dynamical concepts of rational function which can found in $[4,25]$.

We take a square $\mathbb{R} \times \mathbb{R}=[-2,2] \times[-2,2]$ of $256 \times 256$ points and we apply our iterative methods starting in every $z^{(0)}$ in the square. If the sequence generated by the iterative method attempts a zero $z_{j}^{*}$ of the polynomial with a tolerance $\left|f\left(z^{(k)}\right)\right|<\times 10^{-4}$ and a maximum of 100 iterations, we decide that $z^{(0)}$ is in the basin of attraction of this zero. If the iterative method starting in $z^{(0)}$ reaches a zero in $N$ iterations $(N \leq 100)$, then we mark this point $z^{(0)}$ with colors if $\left|z^{(N)}-z_{j}^{*}\right|<\times 10^{-4}$. If $N>50$, we conclude that the starting point has diverged and we assign a dark blue color. Let $N_{D}$ be a number of diverging points and we count the number of starting points which converge in 1, 2, 3, 4, 5 or above 5 iterations. In the following, we describe the basins of attraction for Newton's method and some higher order Newton type methods for finding complex roots of polynomials $p_{1}(z)=z^{2}-1$, $p_{2}(z)=z^{3}-1$ and $p_{3}(z)=z^{5}-1$.
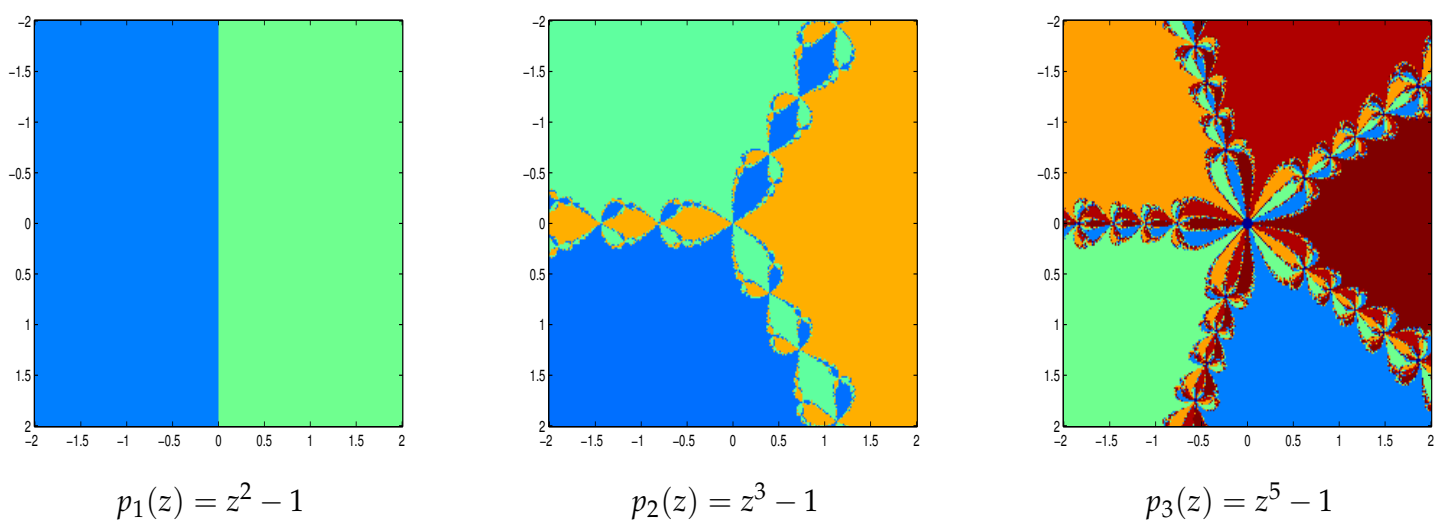

Figure 2. Basins of attraction for $2^{\text {nd }} N R$ for the polynomial $p_{1}(z), p_{2}(z), p_{3}(z)$.

Figures 2 and 3 shows the polynomiographs of the methods for the polynomial $p_{1}(z)$. We can see that the methods $2^{\text {nd }} \mathrm{NR}, 4^{\text {th }} \mathrm{YM}, 8^{\text {th }} \mathrm{SA} 2$ and $8^{\text {th }} \mathrm{YM}$ performed very nicely. The methods $4^{\text {th }} \mathrm{SB}$, $4^{\text {th }} \mathrm{SJ}, 8^{\text {th }} \mathrm{KT}, 8^{\text {th }} \mathrm{LW}, 8^{\text {th }} \mathrm{PNPD}, 8^{\text {th }} \mathrm{SA} 1,8^{\text {th }} \mathrm{CFGT}$ and $8^{\text {th }} \mathrm{CTV}$ are shows some chaotic behavior near the boundary points. The method $4^{\text {th }}$ CLND have sensitive to the choice of initial guess in this case.

Figures 2 and 4 shows the polynomiographs of the methods for the polynomial $p_{2}(z)$. We can see that the methods $2^{\text {nd }} \mathrm{NR}, 4^{\text {th }} \mathrm{YM}, 8^{\text {th }} \mathrm{SA} 2$ and $8^{\text {th }} \mathrm{YM}$ performed very nicely. The methods $4^{\text {th }} \mathrm{SB}, 8^{\text {th }} \mathrm{KT}$, $8^{\text {th }} \mathrm{LW}$ and $8^{\text {th }} \mathrm{CTV}$ are shows some chaotic behavior near the boundary points. The methods $4^{\text {th }} \mathrm{CLND}$, $4^{\text {th }}$ SJ, $8^{\text {th }}$ PNPD, $8^{\text {th }}$ SA1, and $8^{\text {th }}$ CFGT have sensitive to the choice of initial guess in this case.

Figures 2 and 5 shows the polynomiographs of the methods for the polynomial $p_{3}(z)$. We can see that the methods $4^{\text {th }} \mathrm{YM}, 8^{\text {th }} \mathrm{SA} 2$ and $8^{\text {th }} \mathrm{YM}$ are shows some chaotic behavior near the boundary points. The methods $2^{\text {td }} \mathrm{NR}, 4^{\text {th }} \mathrm{SB}, 4^{\text {th }} \mathrm{CLND}, 4^{\text {th }} \mathrm{SJ}, 8^{\text {th }} \mathrm{KT}, 8^{\text {th }} \mathrm{LW}, 8^{\text {th }} \mathrm{PNPD}, 8^{\text {th }} \mathrm{SA} 1,8^{\text {th }} \mathrm{CFGT}$ and $8^{\text {th }}$ CTV have sensitive to the choice of initial guess in this case. In Tables $8-10$, we classify the number of converging and diverging grid points for each iterative method. 


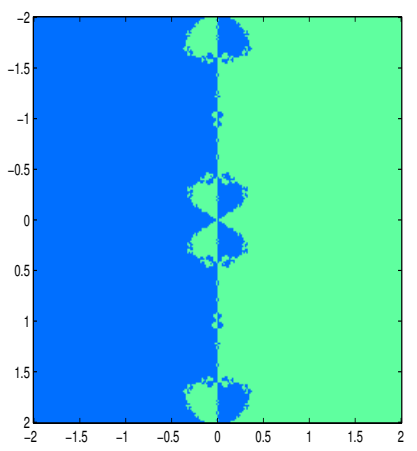

$4^{\text {th }} S B$

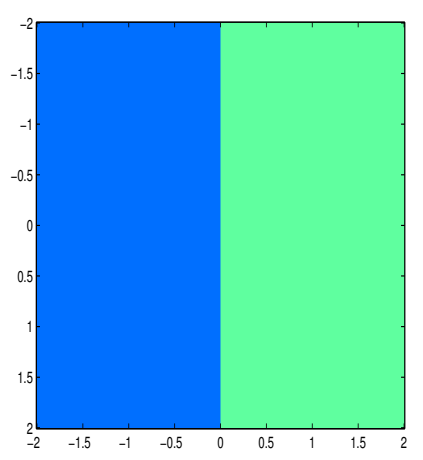

$4^{\text {th }} Y M$

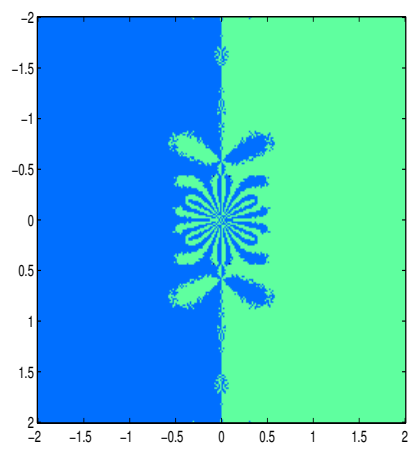

$8^{\text {th }} P N P D$

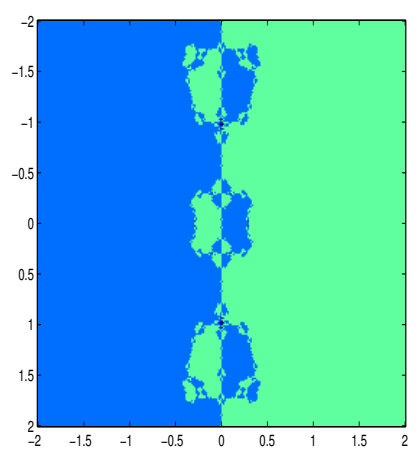

$8^{\text {th }}$ CFGT

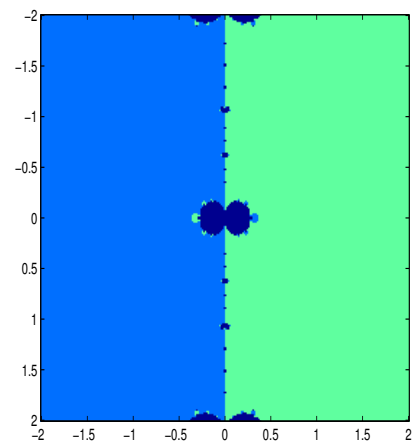

$4^{\text {th }} C L N D$

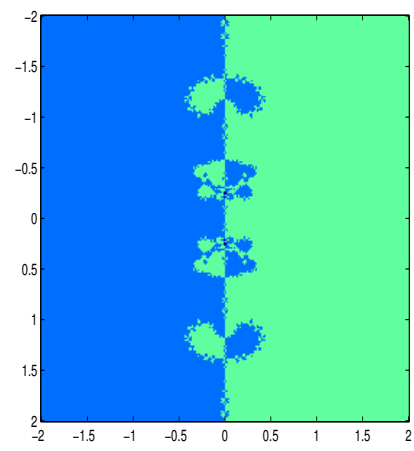

$8^{\text {th }} K T$

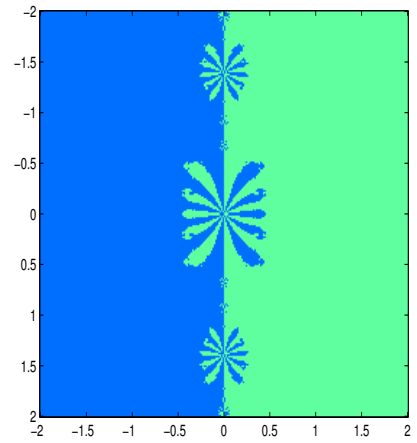

$8^{\text {th }} S A 1$

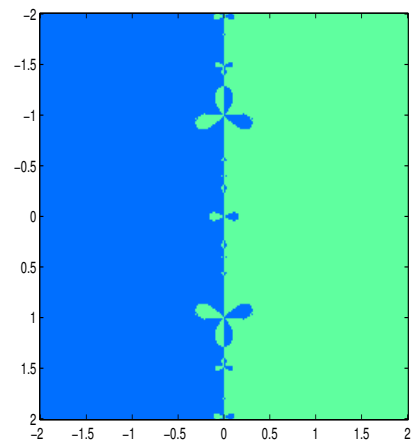

$8^{\text {th }} \mathrm{CTV}$

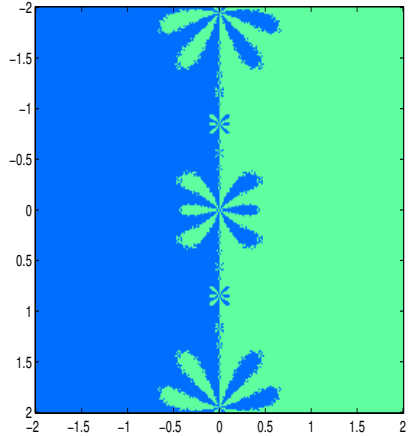

$4^{\text {th }} S J$

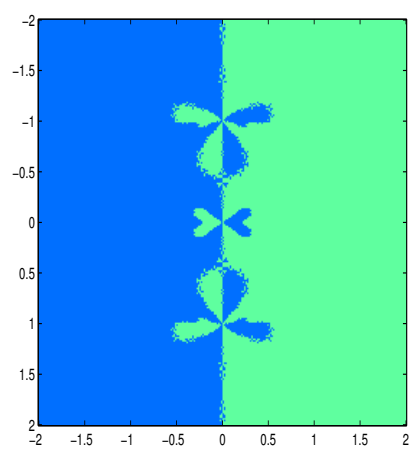

$8^{\text {th }} L W$

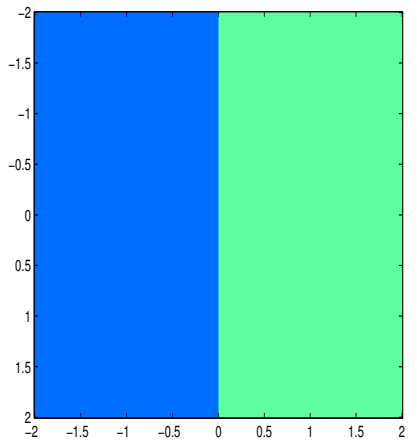

$8^{\text {th }} S A 2$

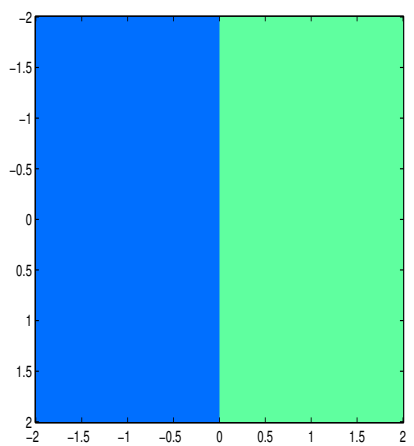

$8^{\text {th }} Y M$

Figure 3. Basins of attraction for $p_{1}(z)=z^{2}-1$. 


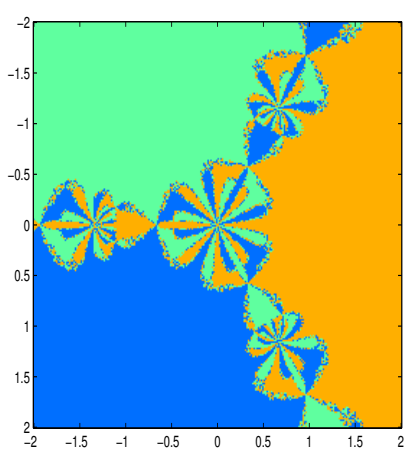

$4^{\text {th }} S B$

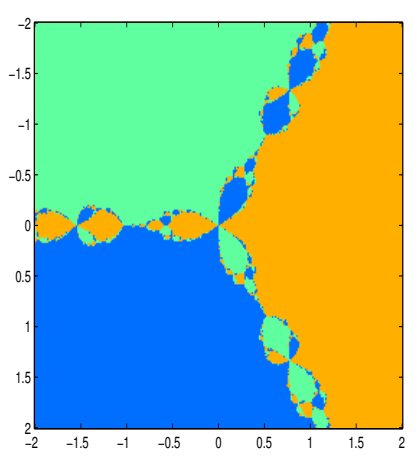

$4^{\text {th }} Y M$

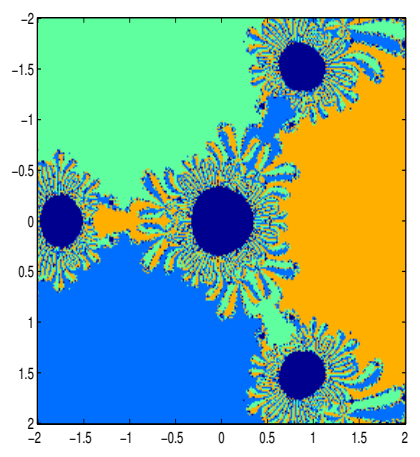

$8^{\text {th }} P N P D$

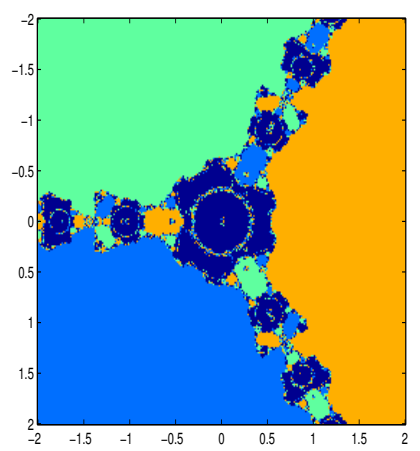

$8^{\text {th }}$ CFGT

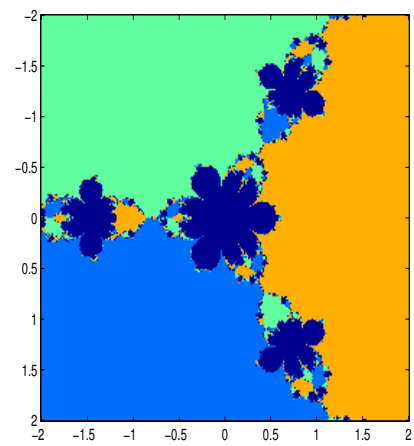

$8^{\text {th }}$ CLND

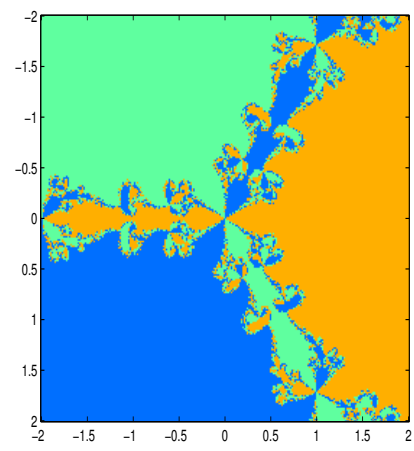

$8^{\text {th }} K T$

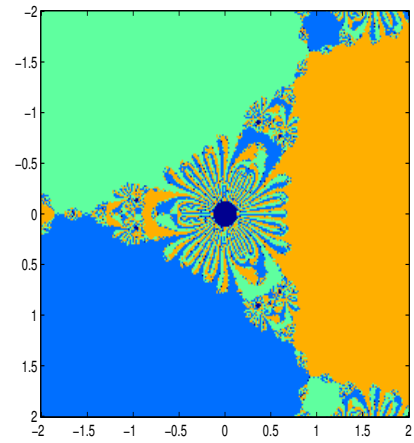

$8^{\text {th }} S A 1$

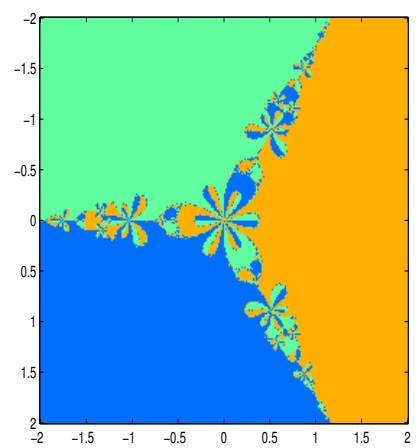

$8^{\text {th }} \mathrm{CTV}$

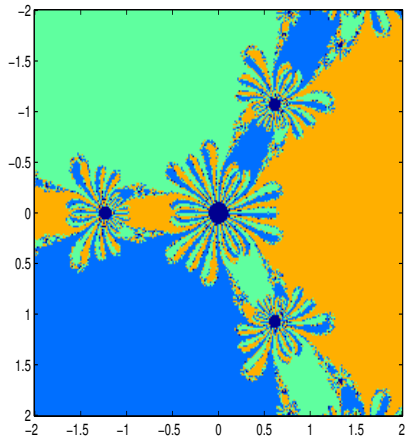

$8^{\text {th }} S J$

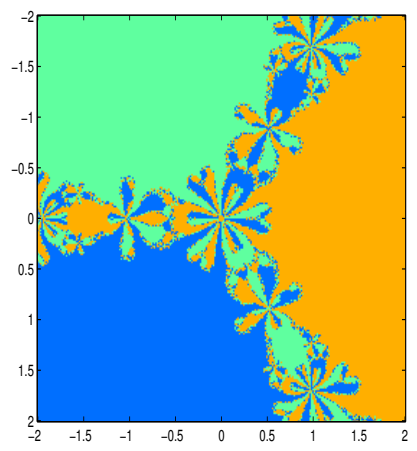

$8^{\text {th }} \mathrm{LW}$

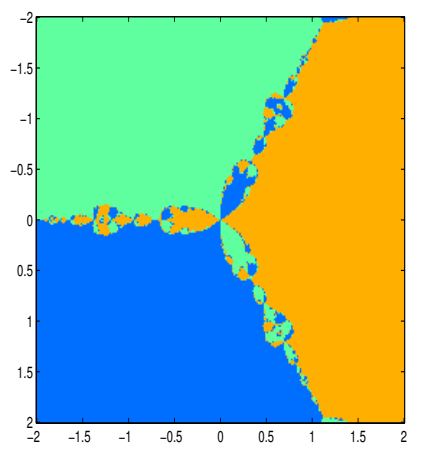

$8^{\text {th }} S A 2$

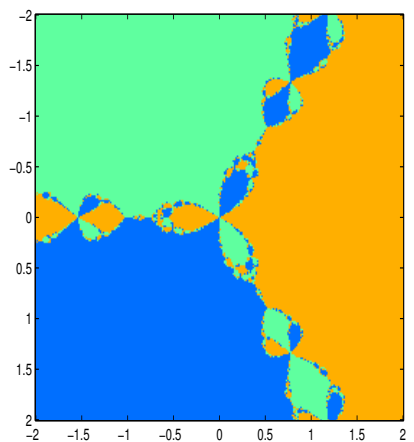

$8^{\text {th }} Y M$

Figure 4. Basins of attraction for $p_{2}(z)=z^{3}-1$. 


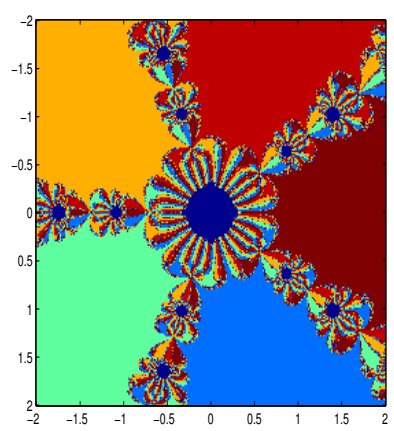

$4^{\text {th }} S B$

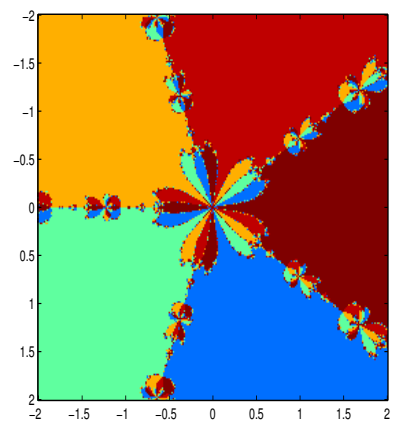

$4^{\text {th }} Y M$

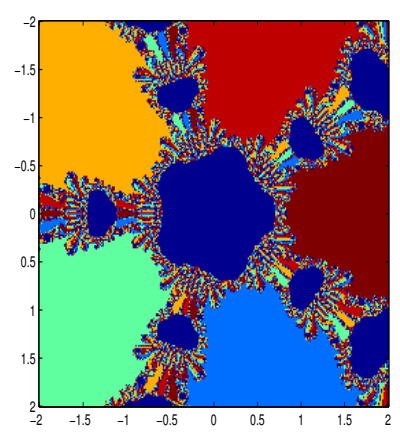

$8^{\text {th }} P N P D$

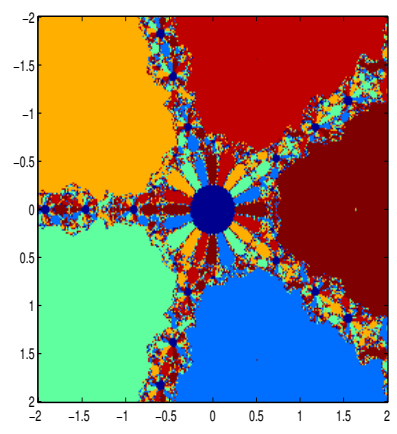

$8^{\text {th }} C F G T$

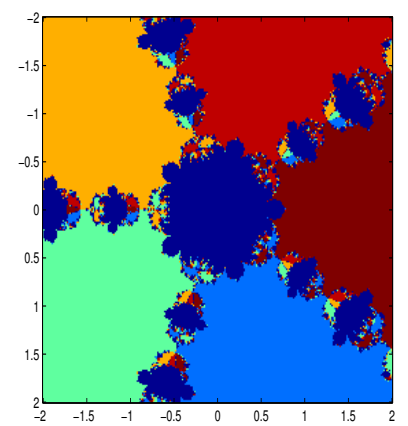

$4^{\text {th }}$ CLND

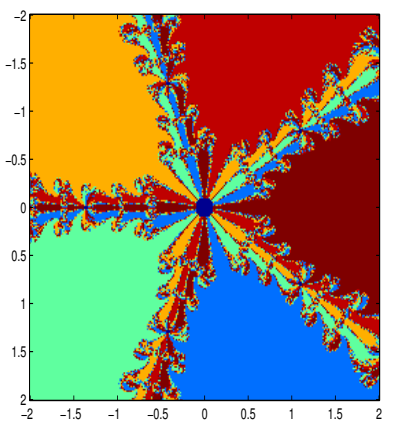

$8^{\text {th }} K T$

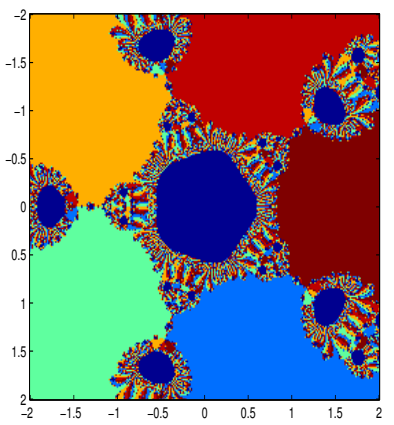

$8^{\text {th }} S A 1$

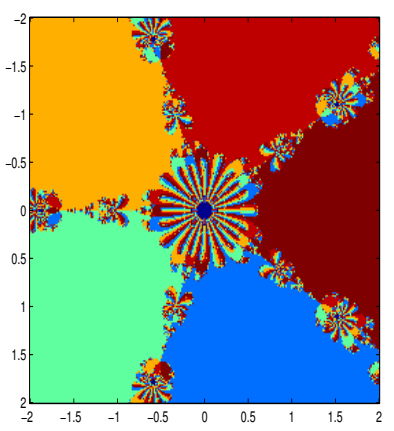

$8^{\text {th }} \mathrm{CTV}$

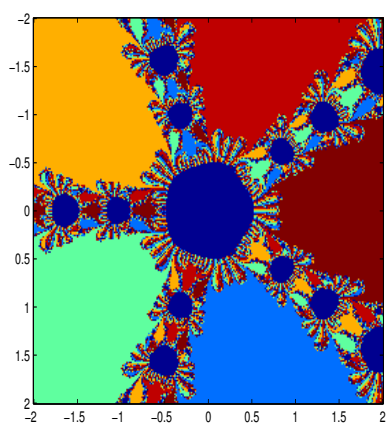

$4^{\text {th }} S J$

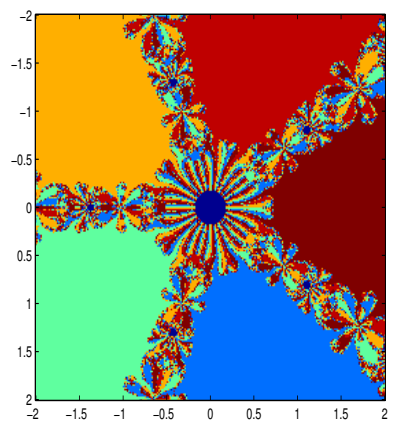

$8^{\text {th }} L W$

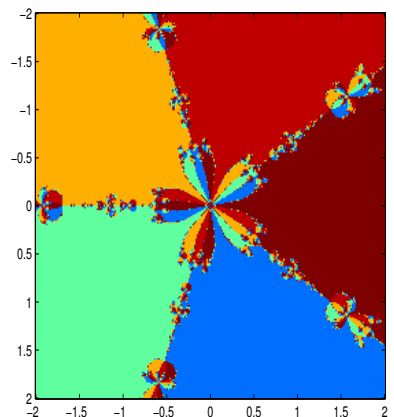

$8^{\text {th }} S A 2$

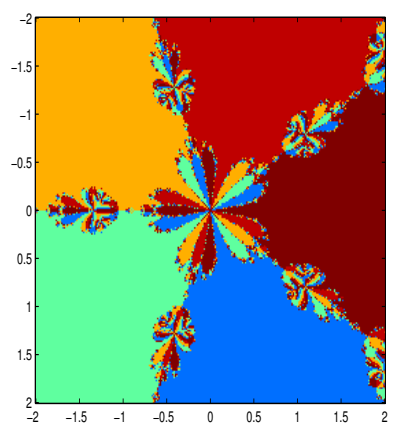

$8^{\text {th }} Y M$

Figure 5. Basins of attraction for $p_{3}(z)=z^{5}-1$. 
Table 8. Results of the polynomials $p_{1}(z)=z^{2}-1$.

\begin{tabular}{cccccccc}
\hline IF & $\boldsymbol{N}=\mathbf{1}$ & $\boldsymbol{N}=\mathbf{2}$ & $\boldsymbol{N}=\mathbf{3}$ & $\mathbf{N = 4}$ & $\boldsymbol{N}=\mathbf{5}$ & $\boldsymbol{N}>\mathbf{5}$ & $\boldsymbol{N}_{\boldsymbol{D}}$ \\
\hline $2^{\text {nd }} \mathrm{NR}$ & 4 & 516 & 7828 & 23,272 & 20,548 & 13,368 & 0 \\
$4^{\text {th }} \mathrm{SB}$ & 340 & 22,784 & 29,056 & 6836 & 2928 & 3592 & 0 \\
$4^{\text {th }} \mathrm{CLND}$ & 372 & 24,600 & 29,140 & 6512 & 2224 & 2688 & 1076 \\
$4^{\text {th }} \mathrm{SJ}$ & 300 & 19,816 & 28,008 & 5844 & 2968 & 8600 & 0 \\
$4^{\text {th }} \mathrm{YM}$ & 520 & 31,100 & 27,520 & 4828 & 1208 & 360 & 0 \\
$8^{\text {th }} \mathrm{KT}$ & 4684 & 44,528 & 9840 & 3820 & 1408 & 1256 & 24 \\
$8^{\text {th }} \mathrm{LW}$ & 4452 & 43,236 & 11,408 & 3520 & 1540 & 1380 & 0 \\
$8^{\text {th }} \mathrm{PNPD}$ & 2732 & 39,768 & 13,112 & 3480 & 1568 & 4876 & 16 \\
$8^{\text {th }} \mathrm{SA} 1$ & 4328 & 45,824 & 8136 & 2564 & 1484 & 3200 & 0 \\
$8^{\text {th }} \mathrm{SA2}$ & 15,680 & 45,784 & 3696 & 376 & 0 & 0 & 0 \\
$8^{\text {th }} \mathrm{CFGT}$ & 9616 & 43,716 & 7744 & 2916 & 980 & 564 & 64 \\
$8^{\text {th }} \mathrm{CTV}$ & 7124 & 48,232 & 7464 & 1892 & 632 & 192 & 0 \\
$8^{\text {th }} \mathrm{YM}$ & 8348 & 50,792 & 5572 & 824 & 0 & 0 & 0 \\
\hline
\end{tabular}

Table 9. Results of the polynomials $p_{2}(z)=z^{3}-1$.

\begin{tabular}{cccccccc}
\hline IF & $N=\mathbf{1}$ & $\boldsymbol{N}=\mathbf{2}$ & $\mathbf{N = 3}$ & $\mathbf{N = 4}$ & $\mathbf{N = 5}$ & $\boldsymbol{N}>\mathbf{5}$ & $\boldsymbol{N}_{\boldsymbol{D}}$ \\
\hline $2^{\text {nd }} \mathrm{NR}$ & 0 & 224 & 2908 & 11,302 & 19,170 & 31,932 & 0 \\
$4^{\text {th }} \mathrm{SB}$ & 160 & 9816 & 27,438 & 9346 & 5452 & 13,324 & 6 \\
$4^{\text {th }} \mathrm{CLND}$ & 170 & 11,242 & 28,610 & 9984 & 4202 & 11,328 & 7176 \\
$4^{\text {th }} \mathrm{SJ}$ & 138 & 7760 & 25,092 & 8260 & 5058 & 19,228 & 1576 \\
$4^{\text {th }} \mathrm{YM}$ & 270 & 18,064 & 30,374 & 9862 & 3688 & 3278 & 0 \\
$8^{\text {th }} \mathrm{KT}$ & 2066 & 34,248 & 11,752 & 6130 & 4478 & 6862 & 0 \\
$8^{\text {th }} \mathrm{LW}$ & 2092 & 33,968 & 12,180 & 4830 & 3030 & 9436 & 0 \\
$8^{\text {th }} \mathrm{PNPD}$ & 1106 & 25,712 & 11,258 & 3854 & 1906 & 21,700 & 10,452 \\
$8^{\text {th }} \mathrm{SA} 1$ & 1608 & 36,488 & 12,486 & 3718 & 1780 & 9456 & 872 \\
$8^{\text {th }} \mathrm{SA2}$ & 6432 & 46,850 & 9120 & 2230 & 640 & 264 & 0 \\
$8^{\text {th }} \mathrm{CFGT}$ & 3688 & 40,740 & 13,696 & 4278 & 1390 & 1744 & 7395 \\
$8^{\text {th }} \mathrm{CTV}$ & 3530 & 43,554 & 11,724 & 3220 & 1412 & 2096 & 0 \\
$8^{\text {th } \mathrm{YM}}$ & 3816 & 43,596 & 12,464 & 3636 & 1302 & 722 & 0 \\
\hline
\end{tabular}

Table 10. Results of the polynomials $p_{3}(z)=z^{5}-1$.

\begin{tabular}{cccccccc}
\hline IF & $N=\mathbf{1}$ & $N=\mathbf{2}$ & $\boldsymbol{N}=\mathbf{3}$ & $\boldsymbol{N}=\mathbf{4}$ & $\mathbf{N = 5}$ & $\boldsymbol{N}>\mathbf{5}$ & $\boldsymbol{N}_{\boldsymbol{D}}$ \\
\hline $2^{\text {nd }} \mathrm{NR}$ & 2 & 100 & 1222 & 4106 & 7918 & 52,188 & 638 \\
$4^{\text {th }} \mathrm{SB}$ & 76 & 3850 & 15,458 & 18,026 & 5532 & 22,594 & 5324 \\
$4^{\text {th }} \mathrm{CLND}$ & 86 & 4476 & 18,150 & 17,774 & 5434 & 19,616 & 12,208 \\
$4^{\text {th }} \mathrm{SJ}$ & 62 & 3094 & 11,716 & 16,840 & 5682 & 28,142 & 19,900 \\
$4^{\text {th }} \mathrm{YM}$ & 142 & 7956 & 27,428 & 15,850 & 5726 & 8434 & 0 \\
$8^{\text {th }} \mathrm{KT}$ & 950 & 17,884 & 20,892 & 5675 & 4024 & 16,111 & 217 \\
$8^{\text {th }} \mathrm{LW}$ & 1032 & 18,764 & 20,622 & 5056 & 3446 & 16,616 & 1684 \\
$8^{\text {th }} \mathrm{PNPD}$ & 496 & 12,770 & 21,472 & 6576 & 2434 & 21,788 & 14,236 \\
$8^{\text {th }} \mathrm{SA} 1$ & 692 & 26,212 & 15,024 & 4060 & 1834 & 17,714 & 8814 \\
$8^{\text {th }} \mathrm{SA2}$ & 2662 & 41,400 & 12,914 & 4364 & 1892 & 2304 & 0 \\
$8^{\text {th }} \mathrm{CFGT}$ & 2008 & 21,194 & 23,734 & 6180 & 3958 & 8462 & 1953 \\
$8^{\text {th }} \mathrm{CTV}$ & 1802 & 36,630 & 13,222 & 4112 & 2096 & 7674 & 350 \\
$8^{\text {th }} \mathrm{YM}$ & 1736 & 27,808 & 21,136 & 5804 & 2704 & 6348 & 0 \\
\hline
\end{tabular}

We note that a point $z_{0}$ belongs to the Julia set if and only if the dynamics in a neighborhood of $z_{0}$ displays sensitive dependence on the initial conditions, so that nearby initial conditions lead to wildly different behavior after a number of iterations. For this reason, some of the methods are getting divergent points. The common boundaries of these basins of attraction constitute the Julia set of the 
iteration function. It is clear that one has to use quantitative measures to distinguish between the methods, since we have a different conclusion when just viewing the basins of attraction.

In order to summarize the results, we have compared mean number of iteration and total number of functional evaluations (TNFE) for each polynomials and each methods in Table 11. The best method based on the comparison in Table 11 is $8^{\text {th }} \mathrm{SA}$. The method with the fewest number of functional evaluations per point is $8^{\text {th }} \mathrm{SA} 2$ followed closely by $4^{\text {th }} \mathrm{YM}$. The fastest method is $8^{\text {th }} \mathrm{SA} 2$ followed closely by $8^{\text {th }} \mathrm{YM}$. The method with highest number of functional evaluation and slowest method is $8^{\text {th }}$ PNPD.

Table 11. Mean number of iteration $\left(N_{\mu}\right)$ and TNFE for each polynomials and each methods.

\begin{tabular}{cccccc}
\hline IF & $N_{\mu}$ for $p_{\mathbf{1}}(z)$ & $N_{\mu}$ for $p_{\mathbf{2}}(z)$ & $N_{\mu}$ for $p_{\mathbf{3}}(z)$ & Average & TNFE \\
\hline $2^{\text {nd }} \mathrm{NR}$ & 4.7767 & 6.4317 & 9.8531 & 7.0205 & 14.0410 \\
$4^{\text {th }} \mathrm{SB}$ & 3.0701 & 4.5733 & 9.2701 & 5.6378 & 16.9135 \\
$4^{\text {th }} \mathrm{CLND}$ & 3.6644 & 8.6354 & 12.8612 & 8.3870 & 25.1610 \\
$4^{\text {th }} \mathrm{SJ}$ & 3.7002 & 7.0909 & 14.5650 & 8.4520 & 25.3561 \\
$4^{\text {th }} \mathrm{YM}$ & 2.6366 & 3.1733 & 4.0183 & 3.2760 & 9.8282 \\
$8^{\text {th }} \mathrm{KT}$ & 2.3647 & 3.1270 & 4.4501 & 3.3139 & 13.2557 \\
$8^{\text {th }} \mathrm{LW}$ & 2.3879 & 3.5209 & 6.3296 & 4.0794 & 16.3178 \\
$8^{\text {th }} \mathrm{PNPD}$ & 2.9959 & 10.5024 & 12.3360 & 8.6114 & 34.4457 \\
$8^{\text {th }} \mathrm{SA} 1$ & 2.5097 & 4.5787 & 9.7899 & 5.6262 & 22.5044 \\
$8^{\text {th }} \mathrm{SA} 2$ & 1.8286 & 2.1559 & 2.5732 & 2.1859 & 8.7436 \\
$8^{\text {th }} \mathrm{CFGT}$ & 2.1683 & 2.8029 & 3.4959 & 2.8223 & 11.2894 \\
$8^{\text {th }} \mathrm{CTV}$ & 2.1047 & 2.4708 & 3.9573 & 2.8442 & 11.3770 \\
$8^{\text {th }} \mathrm{YM}$ & 1.9828 & 2.3532 & 3.3617 & 2.5659 & 10.2636 \\
\hline
\end{tabular}

\section{Concluding Remarks and Future Work}

In this work, we have developed optimal fourth, eighth and sixteenth order iterative methods for solving nonlinear equations using the divided difference approximation. The methods require the computations of three functions evaluations reaching order of convergence is four, four functions evaluations reaching order of convergence is eight and five functions evaluations reaching order of convergence is sixteen. In the sense of convergence analysis and numerical examples, the Kung-Traub's conjecture is satisfied. We have tested some examples using the proposed schemes and some known schemes, which illustrate the superiority of the proposed method $16^{\text {th }} \mathrm{YM}$. Also, proposed methods and some existing methods have been applied on the Projectile motion problem and Planck's radiation law problem. The results obtained are interesting and encouraging for the new method $16^{\text {th }} \mathrm{YM}$. The numerical experiments suggests that the new methods would be valuable alternative for solving nonlinear equations. Finally, we have also compared the basins of attraction of various fourth and eighth order methods in the complex plane.

Future work includes:

- Now we are investigating the proposed scheme to develop optimal methods of arbitrarily high order with Newton's method, as in [26].

- Also, we are investigating to develop derivative free methods to study dynamical behavior and local convergence, as in $[27,28]$.

Author Contributions: The contributions of both the authors have been similar. Both of them have worked together to develop the present manuscript.

Funding: This paper is supported by three project funds: 1. National College Students Innovation and entrepreneurship training program of Ministry of Education of the People's Republic of China in 2017: Internet Animation Company in Minority Areas-Research Model of “Building Dream" Animation Company. (Project number: 201710684001). 2. Yunnan Provincial Science and Technology Plan Project University Joint Project 2017: Research on Boolean Satisfiability Dividing and Judging Method Based on Clustering and Partitioning (Project number: 2017FH001-056). 3. Qujing Normal college scientific research fund special project (Project number: 2018zx003). 
Acknowledgments: The authors would like to thank the editors and referees for the valuable comments and for the suggestions to improve the readability of the paper.

Conflicts of Interest: The authors declare no conflict of interest.

\section{References}

1. Ostrowski, A.M. Solutions of Equations and System of Equations; Academic Press: New York, NY, USA, 1960.

2. Kung, H.T.; Traub, J.F. Optimal order of one-point and multipoint iteration. J. Assoc. Comput. Mach. 1974, 21, 643-651.

3. Amat, S.; Busquier, S.; Plaza, S. Dynamics of a family of third-order iterative methods that do not require using second derivatives. Appl. Math. Comput. 2004, 154, 735-746.

4. Amat, S.; Busquier, S.; Plaza, S. Review of some iterative root-finding methods from a dynamical point of view. Scientia 2004, 10, 3-35.

5. Babajee, D.K.R; Madhu, K.; Jayaraman, J. A family of higher order multi-point iterative methods based on power mean for solving nonlinear equations. Afrika Matematika 2016, 27, 865-876. [CrossRef]

6. Cordero, A.; Fardi, M.; Ghasemi, M.; Torregrosa, J.R. Accelerated iterative methods for finding solutions of nonlinear equations and their dynamical behavior. Calcolo 2014, 51, 17-30.

7. Cordero, A.; Torregrosa, J.R.; Vasileva, M.P. A family of modified ostrowski's methods with optimal eighth order of convergence. Appl. Math. Lett. 2011, 24, 2082-2086.

8. Liu, L.; Wang, X. Eighth-order methods with high efficiency index for solving nonlinear equations. Appl. Math. Comput. 2010, 215, 3449-3454.

9. Madhu, K. Some New Higher Order Multi-Point Iterative Methods and Their Applications to Differential and Integral Equation and Global Positioning System. Ph.D. Thesis, Pndicherry University, Kalapet, India, June 2016.

10. Madhu, K.; Jayaraman, J. Higher order methods for nonlinear equations and their basins of attraction. Mathematics 2016, 4, 22.

11. Petkovic, M.S.; Neta, B.; Petkovic, L.D.; Dzunic, J. Multipoint Methods for Solving Nonlinear Equations; Elsevier: Amsterdam, The Netherlands, 2012.

12. Sharma, J.R.; Arora, H. An efficient family of weighted-newton methods with optimal eighth order convergence. Appl. Math. Lett. 2014, 29, 1-6.

13. Sharma, J.R.; Arora, H. A new family of optimal eighth order methods with dynamics for nonlinear equations. Appl. Math. Comput. 2016, 273, 924-933.

14. Soleymani, F.; Khratti, S.K.; Vanani, S.K. Two new classes of optimal Jarratt-type fourth-order methods. Appl. Math. Lett. 2011, 25, 847-853.

15. Wait, R. The Numerical Solution of Algebraic Equations; John Wiley and Sons: Hoboken, NJ, USA, 1979.

16. Khan, Y.; Fardi, M.; Sayevand, K. A new general eighth-order family of iterative methods for solving nonlinear equations. Appl. Math. Lett. 2012, 25, 2262-2266.

17. Cordero, A.; Torregrosa, J.R. Variants of Newton's method using fifth-order quadrature formulas. Appl. Math. Comput. 2007, 190, 686-698.

18. Sharma, R.; Bahl, A. An optimal fourth order iterative method for solving nonlinear equations and its dynamics. J. Complex Anal. 2015, 2015, 259167.

19. Chun, C; Lee, M. Y.; Neta, B; Dzunic, J. On optimal fourth-order iterative methods free from second derivative and their dynamics. Appl. Math. Comput. 2012, 218, 6427-6438. [CrossRef]

20. Singh, A.; Jaiswal, J.P. Several new third-order and fourth-order iterative methods for solving nonlinear equations. Int. J. Eng. Math. 2014, 2014, 828409.

21. Babajee, D.K.R.; Madhu, K. Comparing two techniques for developing higher order two-point iterative methods for solving quadratic equations. SeMA J. 2018, 1-22. [CrossRef]

22. Kantrowitz, R.; Neumann, M.M. Some real analysis behind optimization of projectile motion. Mediterr. J. Math. 2014, 11, 1081-1097.

23. Henelsmith, N. Finding the Optimal Launch Angle; Whitman College: Walla Walla, WA, USA, 2016.

24. Bradie, B. A Friendly Introduction to Numerical Analysis; Pearson Education Inc.: New Delhi, India, 2006.

25. Scott, M.; Neta, B.; Chun, C. Basin attractors for various methods. Appl. Math. Comput. 2011, 218, $2584-2599$. 
26. Cordero, A.; Hueso, J. L.; Martinez, E.; Torregrosa, J.R. Generating optimal derivative free iterative methods for nonlinear equations by using polynomial interpolation. Appl. Math. Comput. 2013, 57 1950-1956. [CrossRef]

27. Argyros, I.K.; Magrenan, A.A.; Orcos, L. Local convergence and a chemical application of derivative free root finding methods with one parameter based on interpolation. J. Math. Chem. 2016, 54, 1404-1416.

28. Zafar, F.; Cordero, A.; Torregrosa, J.R. An efficient family of optimal eighth-order multiple root finders. Mathematics 2018, 6, 310 .

(C) 2019 by the authors. Licensee MDPI, Basel, Switzerland. This article is an open access article distributed under the terms and conditions of the Creative Commons Attribution (CC BY) license (http://creativecommons.org/licenses/by/4.0/). 